\title{
Mitochondrial proteolytic stress induced by loss of mortalin function is rescued by Parkin and PINK1
}

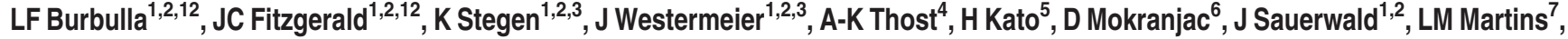 \\ D Woitalla ${ }^{8}$, D Rapaport ${ }^{5}$, 0 Riess $^{9}$, T Proikas-Cezanne ${ }^{4}$, TM Rasse ${ }^{\star, 10}$ and R Krüger ${ }^{\star, 1,2,11}$
}

The mitochondrial chaperone mortalin was implicated in Parkinson's disease (PD) because of its reduced levels in the brains of PD patients and disease-associated rare genetic variants that failed to rescue impaired mitochondrial integrity in cellular knockdown models. To uncover the molecular mechanisms underlying mortalin-related neurodegeneration, we dissected the cellular surveillance mechanisms related to mitochondrial quality control, defined the effects of reduced mortalin function at the molecular and cellular levels and investigated the functional interaction of mortalin with Parkin and PINK1, two PD-related proteins involved in mitochondrial homeostasis. We found that reduced mortalin function leads to: (1) activation of the mitochondrial unfolded protein response (UPR(mt)), (2) increased susceptibility towards intramitochondrial proteolytic stress, (3) increased autophagic degradation of fragmented mitochondria and (4) reduced mitochondrial mass in human cells in vitro and ex vivo. These alterations caused increased vulnerability toward apoptotic cell death. Proteotoxic perturbations induced by either partial loss of mortalin or chemical induction were rescued by complementation with native mortalin, but not diseaseassociated mortalin variants, and were independent of the integrity of autophagic pathways. However, Parkin and PINK1 rescued loss of mortalin phenotypes via increased lysosomal-mediated mitochondrial clearance and required intact autophagic machinery. Our results on loss of mortalin function reveal a direct link between impaired mitochondrial proteostasis, UPR(mt) and PD and show that effective removal of dysfunctional mitochondria via either genetic (PINK1 and Parkin overexpression) or pharmacological intervention (rapamycin) may compensate mitochondrial phenotypes.

Cell Death and Disease (2014) 5, e1180; doi:10.1038/cddis.2014.103; published online 17 April 2014

Subject Category: Neuroscience

Although 18 genetic disease loci and several environmental factors have been identified (reviewed in Sharma et al. ${ }^{1}$ ) that contribute to Parkinson's disease (PD), the exact pathogenic mechanisms remain elusive. ${ }^{2}$ Neuronal function critically relies on intact mitochondrial homeostasis. Impaired mitochondrial function and dynamics alter neuronal bioenergetics, an underlying factor in aging and a number of neurodegenerative diseases, leading to programmed cell death., ${ }^{3,4}$

Several PD-associated genes such as PINK1, Parkin or $D J-1$ encode proteins that are closely linked to mitochondrial quality control, thus providing an important molecular link between mitochondrial homeostasis and neurodegeneration observed in PD. Disease-related mutations in these genes cause loss of protein function and contribute to impaired mitochondrial integrity as revealed by the loss of mitochondrial membrane potential (MMP), disrupted mitochondrial morphology and reduced activity of complex I of the electron transport chain. ${ }^{5-7}$

Unbiased biochemical approaches identified the mitochondrial stress response protein mortalin (also known as glucose regulated protein 75 (GRP75) or mitochondrial heat shock protein $70(\mathrm{mtHsp} 70))$ as an interactor of Parkin, PINK1 and DJ-1. ${ }^{8-11}$ Mortalin is a member of the Hsp70 family and was identified as a molecular chaperone within the mitochondrial

\footnotetext{
${ }^{1}$ German Center for Neurodegenerative Diseases, Tübingen, Germany; ${ }^{2}$ Department of Neurodegenerative Diseases and Hertie-Institute for Clinical Brain Research, University of Tübingen, Tübingen, Germany; ${ }^{3}$ Graduate School of Cellular and Molecular Neuroscience, University of Tübingen, Tübingen, Germany; ${ }^{4}$ Autophagy Laboratory, Interfaculty Institute for Cell Biology, University of Tübingen, Tübingen, Germany; ${ }^{5}$ Interfaculty Institute of Biochemistry, University of Tübingen, Tübingen, Germany; ${ }^{6}$ Adolf Butenandt Institute, Physiological Chemistry, University of Munich, Munich, Germany; ${ }^{7}$ Cell Death Regulation Laboratory, MRC Toxicology Unit, Leicester, UK; ${ }^{8}$ Department of Neurology, St. Josef-Hospital, Ruhr-University Bochum, Bochum, Germany; ${ }^{9}$ Medical Genetics, University of Tübingen, Tübingen, Germany; ${ }^{10}$ Junior Research Group Synaptic Plasticity, Hertie-Institute for Clinical Brain Research, University of Tübingen, Tübingen, Germany and ${ }^{11}$ Werner Reichardt Centre for Integrative Neuroscience, University of Tübingen, Tübingen, Germany

${ }^{*}$ Corresponding authors: TM Rasse, Junior Research Group Synaptic Plasticity, Hertie-Institute for Clinical Brain Research, University of Tübingen, Otfried-MüllerStrasse 27, 72076 Tübingen, Germany. Tel: +49 7071 2981948; Fax: +49 7071 294697; E-mail: tobias.rasse@ uni-tuebingen.de

or R Krüger, Laboratory of Functional Neurogenomics, Center of Neurology and Hertie-Institute for Clinical Brain Research, University of Tübingen, and German Center for Neurodegenerative Diseases (DZNE), Otfried-Müller-Strasse 27, 72076 Tübingen, Germany. Tel: +49 70712982141 ; Fax: +49 7071295260 ; E-mail: rejko.krueger@uni-tuebingen.de

${ }^{12}$ These authors contributed equally to this work.

Keywords: Parkinson's disease; mortalin; mitochondrial; proteolytic stress; mitophagy

Abbreviations: 17-AAG, 17-allylamino-17-demethoxygeldanamycin; BafA1, bafilomycin A1; CCCP, carbonyl cyanide m-chlorophenyl hydrazone; CM, control medium condition; dOTC, truncated version of OTC; EBSS, Earle's balanced salt solution; ER, endoplasmic reticulum; GRP75, glucose regulated protein 75; KO, knockout; MEF, mouse embryonic fibroblast; Mfn2, mitofusin 2; MMP, mitochondrial membrane potential; mtHsp70, mitochondrial heat shock protein 70; OTC, ornithine transcarbamylase; PBS, phosphate-buffered saline; PD, Parkinson's disease; Ptdlns(3)P, phosphatidylinositol 3-phosphate; ROS, reactive oxygen species; TG, thapsigargin; UPR(mt), mitochondrial unfolded protein response; Wt, wild type

Received 16.7.13; revised 30.1.14; accepted 14.2.14; Edited by A Verkhratsky
} 
matrix. ${ }^{8,12-14}$ As the only ATPase component of the mitochondrial import complex, mortalin is essential for the effective import and folding of nuclear-encoded mitochondrial matrix proteins as well as for the proper degradation of altered or impaired mitochondrial proteins. ${ }^{15,16}$ Mortalin is a key player in mitochondrial stress response, aging and programmed cell death. ${ }^{17-19}$ Overexpression of mortalin extends lifespan in human cells and the nematode $C$. elegans, indicating a protective function in vitro and in vivo. ${ }^{11,20,21}$ In contrast, partial loss of mortalin results in alterations in mitochondrial morphology, impaired MMP and increased cellular levels of reactive oxygen species (ROS).$^{11}$ Analysis of three PD-associated variants in the mortalin gene revealed a loss of protective mortalin function in human cells. ${ }^{11}$ Notably, mortalin was linked to neurodegeneration in PD based on substantially reduced levels of the protein in brain samples of patients. ${ }^{19,22}$ It was further observed that the reduction in the levels of mortalin in patients correlated with the disease stage. ${ }^{22}$ RNAi-mediated knockdown of mortalin in Drosophila recapitulates defects observed in other invertebrate PD models, reducing cellular ATP levels and inducing defects in body posture and locomotion. ${ }^{23}$ Importantly, loss of synaptic mitochondria, mediated by mitophagy, was observed early in disease progression ${ }^{23}$ and also preceded behavioral impairments and changes in synaptic morphology in other PD-associated Drosophila models. ${ }^{24,25}$

In order to investigate how loss of mortalin function relates to neurodegeneration in PD and influences mechanisms related to molecular and organellar quality control, we studied both in vitro and ex vivo cellular models. Our results reveal a primary defect in intramitochondrial protein quality control because of loss of mortalin associated with an increased mitochondrial unfolded protein response (UPR(mt)) and increased susceptibility of cells toward intramitochondrial proteolytic stress. The proteotoxic perturbations caused by loss of mortalin or chemical induction were rescued by complementation with wild-type (wt) mortalin, but not PD-associated mortalin variants, and were independent of downstream autophagic clearance machinery. Importantly, Parkin and PINK1 rescued loss of mortalin-associated mitochondrial fragmentation and apoptotic cell death via an activation of autophagic clearance of mitochondria. Importantly, a successful rescue was dependent on intact lysosomal degradation pathways.

Collectively, we provide first insights into the role of the intramitochondrial protein quality control in PD and integrate mortalin defects into molecular pathways related to PINK1/ Parkin-mediated organellar homeostasis in PD pathogenesis.

\section{Results}

Loss of mortalin increases intramitochondrial proteolytic stress. As a mitochondrial chaperone in the mitochondrial matrix, mortalin is critically required for the proper import and folding of nuclear-encoded matrix proteins. ${ }^{17} \mathrm{We}$ hypothesized that PD-associated loss of mortalin function initiates impaired mitochondrial protein homeostasis. We first sought to measure the ratio of nuclear-encoded ATP5A to the mitochondrially encoded MTCO1 to assess potential mitonuclear imbalance. Mitonuclear imbalance was recently reported to precede activation of $\operatorname{UPR}(\mathrm{mt})$, together comprising a stress-signaling pathway conserved across many species. ${ }^{26}$ We found reduced mitochondrially encoded MTCO1 protein levels in mortalin knockdown cells compared with controls, whereas the level of nuclear-encoded ATP5A remained the same (Figure 1a). To further investigate the relevance of loss of mortalin to mitochondrial quality control, we measured the levels of the mitochondrial chaperone Hsp60 as a marker for the $\operatorname{UPR}(\mathrm{mt})$ as this protein is upregulated in conditions of intramitochondrial proteolytic stress (reviewed in Broadley and $\mathrm{Hart}^{27}$ ). To assess the intramitochondrial stress response upon loss of mortalin function, we analyzed the Hsp60 protein levels after mortalin knockdown in human neuroblastoma (SH-SY5Y) cells and in human fibroblasts from a heterozygous carrier of the PD-associated A476T mortalin variant (hereafter named A476T-fibroblasts). To validate the efficacy of mortalin knockdown in human neuroblastoma cells, mortalin levels were quantified. Western blot analysis revealed $\sim 70 \%$ reduction in mortalin protein (Supplementary Figure S1a).

Because the abundance of Hsp60 may depend on the mitochondrial mass, we normalized our results to a mitochondrial marker (Tom20). We observed increased levels of Hsp60 in SH-SY5Y cells with reduced mortalin levels (Figure 1b). Similar alterations in Hsp60 levels were found in A476Tfibroblasts compared with control fibroblasts that may reflect a chronic activation of UPR $(\mathrm{mt})$ in mutant cells (Figure 1c). Our results on mitonuclear imbalance and upregulation of the stress protein Hsp60 suggest that loss of mortalin function induces a mitochondrial stress response arising within the mitochondria. We hypothesize that the mitochondrial stress, mitonuclear imbalance and UPR(mt) activation in cells lacking functional mortalin would arise as a result of proteolytic stress. Therefore, we investigated whether mortalin knockdown causes an accumulation of aggregated or insoluble proteins within mitochondria by silver staining. As a result, no differences in the levels of SDS-soluble proteins within the mitochondria of mortalin knockdown or control cells were observed, but a significant increase in the amount of insoluble aggregates from the same mitochondrial sample was observed (Figure 1d). This indicates an increase in aggregated proteins within mitochondria upon loss of mortalin function that might arise from the significant amount of unfolded or misfolded proteins that could not be degraded efficiently.

Activation of the UPR $(\mathrm{mt})$ might sensitize or protectively precondition cells toward a second insult that causes further intramitochondrial stress. To differentiate these two possibilities, we induced intramitochondrial stress genetically by expressing the mitochondrial matrix protein ornithine transcarbamylase (OTC) or its truncated variant (dOTC) harboring a FLAG tag in SH-SY5Y cells. The accumulation of the truncated variant in an unfolded state within the mitochondrial matrix has been previously shown to elicit an intramitochondrial proteolytic stress response paralleled by Hsp60 upregulation. ${ }^{28,29}$ Both native OTC and dOTC were correctly targeted to mitochondria upon overexpression (Figure 2a). As expected, overexpression of the truncated dOTC variant led to a more punctate pattern in both conditions, control as well as mortalin knockdown. Interestingly, we also observed a more punctate pattern after overexpression of native OTC in mortalin knockdown cells 
that was not obvious in controls cells. Therefore, we analyzed the area of mitochondria (Tom20 signal) that colocalized with the area covered by OTC signal as a readout for proper intraorganellar distribution of the protein. The mitochondrial area covered by the OTC signal was significantly reduced in mortalin knockdown cells (Figure $2 \mathrm{~b}$ ). This may indicate an increased tendency of native OTC to aggregate in a mortalin
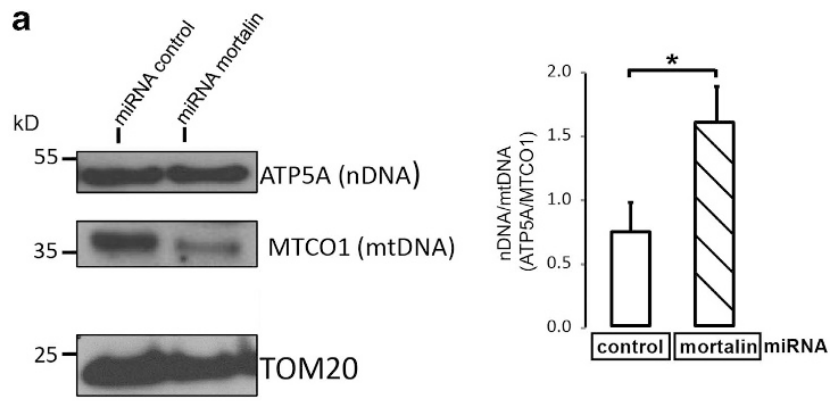

b
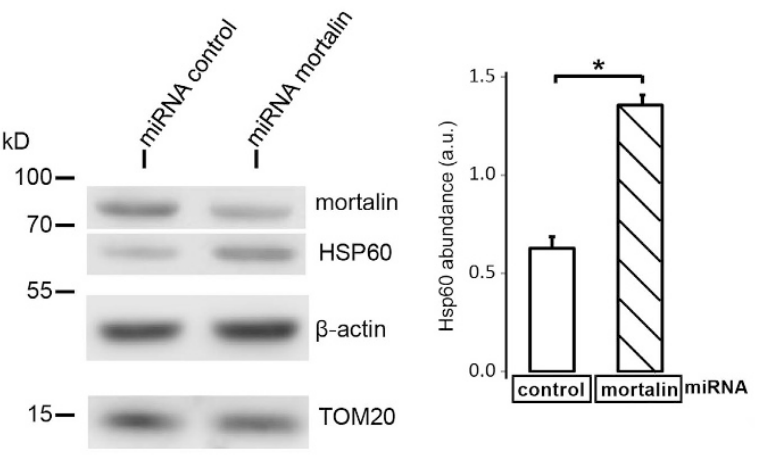

C
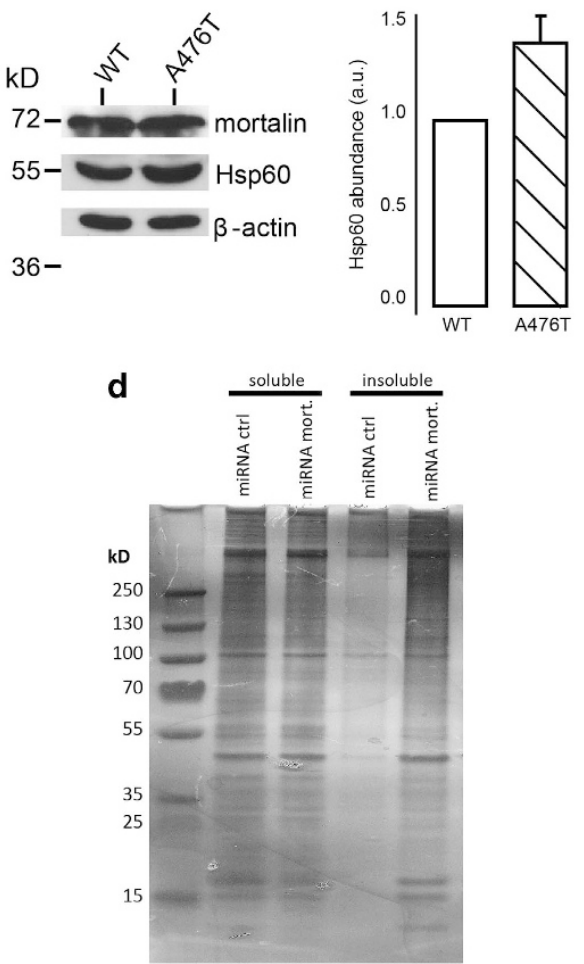

knockdown background and argues in favor of an imbalanced protein folding capacity within the matrix.

Intramitochondrial proteolytic stress due to mortalin knockdown affects mitochondrial integrity similar to dOTC expression. Impaired intramitochondrial protein quality control affects mitochondrial morphology and dynamics. ${ }^{30,31}$ We next extended our previous findings on mitochondrial fragmentation in A476T-fibroblasts ${ }^{11}$ to a human neuronal cell line and confirmed the characteristic fragmentation of the mitochondrial network in response to mortalin knockdown as assessed by aspect ratio, form factor and mitochondrial area per single mitochondrion (mitochondrial size) (Figure 3a). In contrast to wt mortalin, the PDassociated mortalin variants were not able to rescue the disturbed mitochondrial network caused by reduced mortalin function. Next, we used dOTC as a tool to genetically induce intramitochondrial proteolytic stress and then investigated mitochondrial integrity in SH-SY5Y cells with or without mortalin knockdown. Expression of dOTC in SH-SY5Y cells treated with control siRNA resulted in the fragmentation of the mitochondrial network as described previously for nonneuronal human cells ${ }^{28}$ (Figure $3 b$ ). The expression of native OTC in control cells did not affect mitochondrial integrity. Interestingly, overexpression of dOTC in control siRNA-treated cells recapitulated the mitochondrial fragmentation seen in SH-SY5Y cells with reduced mortalin levels. Moreover, expression of dOTC in the mortalin knockdown background further exacerbated the mitochondrial fragmentation phenotype compared with native OTC. The effect of dOTC on mitochondrial integrity is similar in both control and mortalin knockdown cells with no significant synergistic effect

Figure 1 Loss of mortalin evokes a mitochondrial stress response via Hsp60 in human cells. (a, left panel) Western blot analysis of protein levels of the nuclearencoded complex $V \alpha$-subunit (ATP5A) and the mitochondrially encoded complex IV subunit MTCO1 in SH-SY5Y cells transfected with mortalin miRNA and a nontargeting control miRNA (Ctrl). Mitochondrially encoded MTCO1 levels are reduced compared with control, whereas there are no changes in ATP5A and the loading control TOM20. (a, right panel) Quantification by densitometry and calculation of the ATP5A/MTCO1 ratio showed a significantly higher ratio in mortalin knockdown cells indicating mitonuclear imbalance, $n=3$. (b, left panel) Immunoblotting analysis of Hsp60 levels in SH-SY5Y cells. A representative immunoblot shows increased Hsp60 protein levels upon mortalin knockdown. (b, right panel) Densitometric analysis of average Hsp60 levels, normalized to Tom20 levels, $n=3$. (c, left panel) Immunoblotting analysis of Hsp60 levels in human fibroblasts from a carrier of a loss of mortalin variant compared with fibroblasts from a healthy sibling control. A representative immunoblot shows increased Hsp60 protein levels in the A476T fibroblasts. It is noteworthy that no reduction of the overall levels of mortalin can be expected as fibroblasts from a human carrier of the A476T mutation express both the mutant and the wild-type allele. (c, right panel) Densitometric analysis of average Hsp60 levels normalized to $\beta$-actin loading control and expressed as a percentage of control fibroblasts, $n=3$. (d) Silver staining of soluble and insoluble mitochondrial extracts from SH-SY5Y cells transfected with mortalin miRNA and a nontargeting control miRNA (Ctrl). A total of $1 \mu \mathrm{g}$ of total crude mitochondrial extracts were split and incubated in equal volumes of buffer containing $2 \%$ SDS (soluble) and 1\% NP40 (insoluble). Insoluble proteins from mitochondria were isolated from the $1 \%$ NP40 fraction by high-speed centrifugation. Equal volumes of the soluble SDS fraction or the insoluble protein pellet were resolved on NuPAGE $4-12 \%$ gradient gels and subsequent silver staining. Whereas the soluble fraction shows a similar amount of total protein for both miRNAs, the insoluble fraction exhibits an enrichment in 1\% NP40-insoluble protein in the mortalin knockdown condition compared with the control 
a

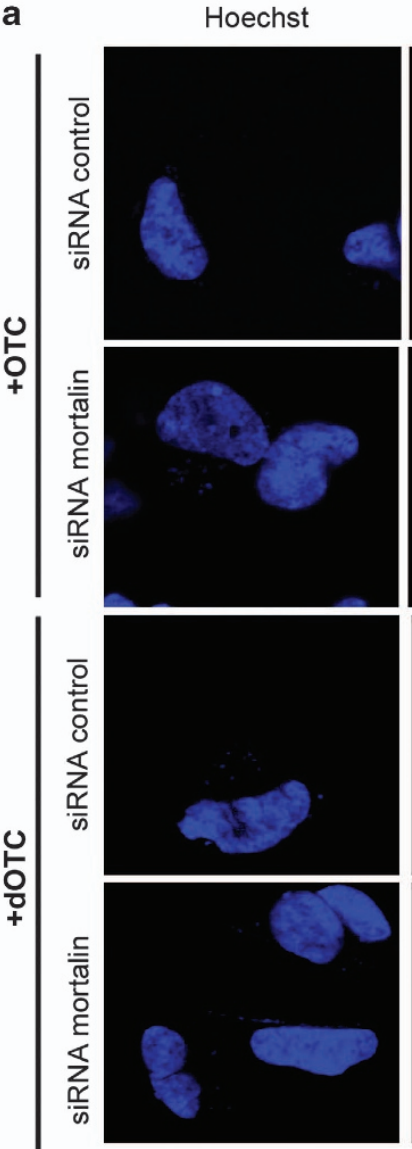

siRNA Alexa555
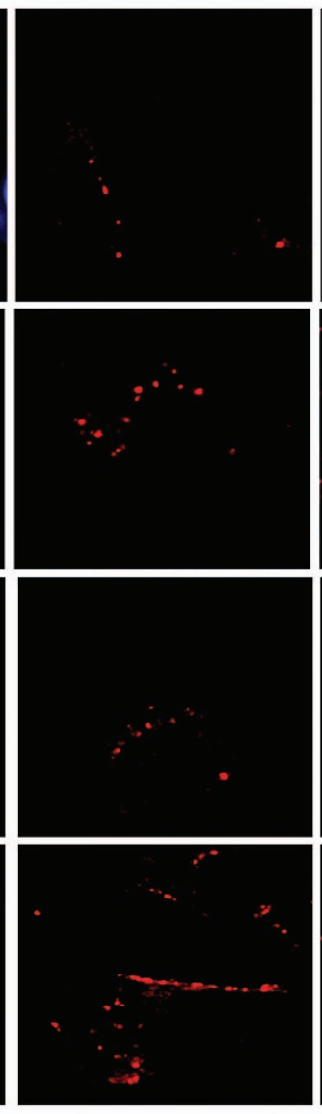

b
TOM20
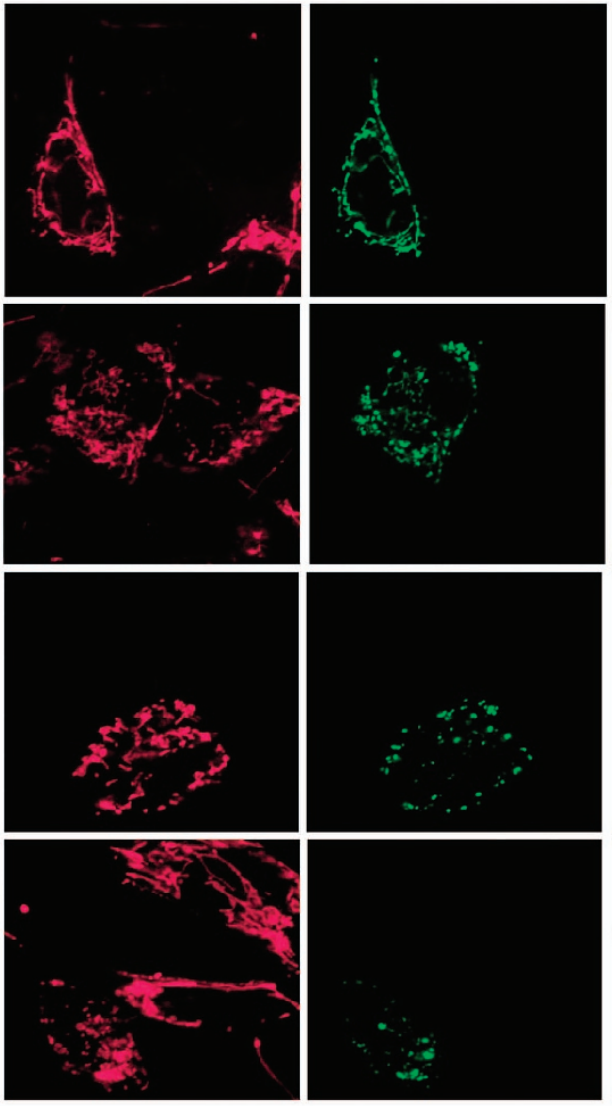

Flag
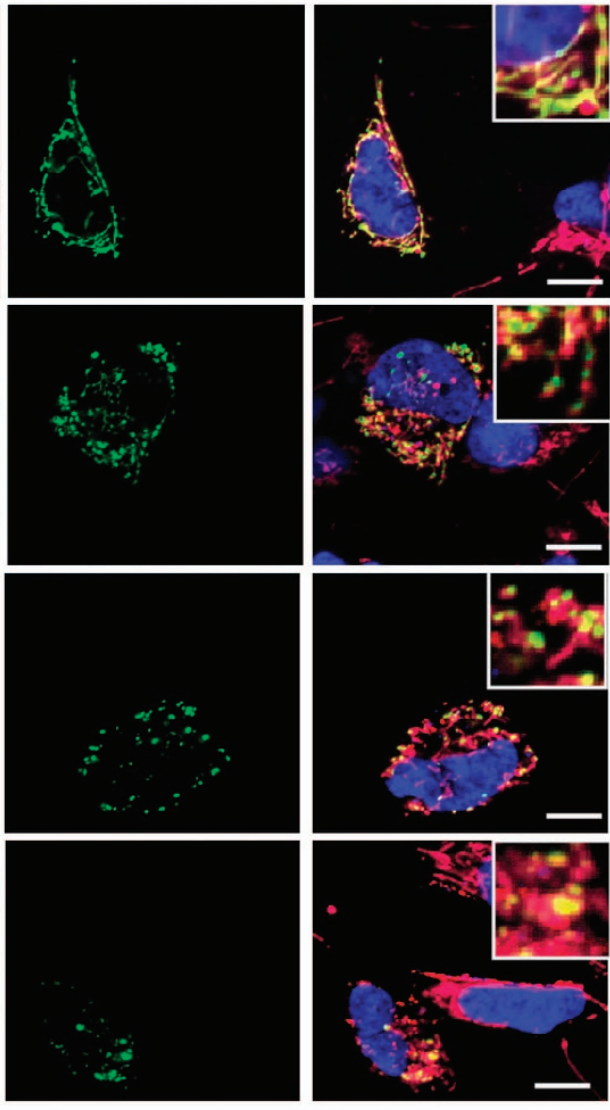

merge
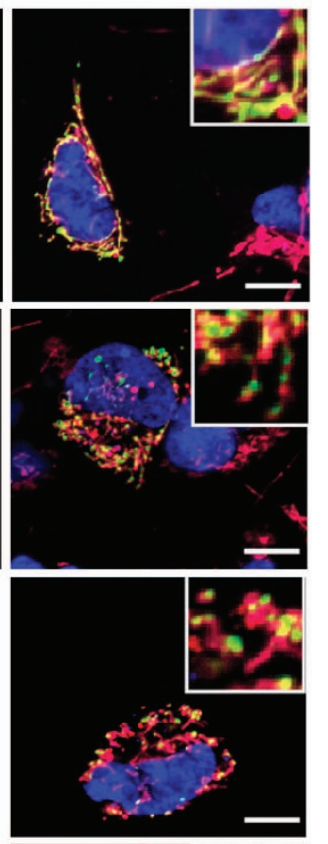

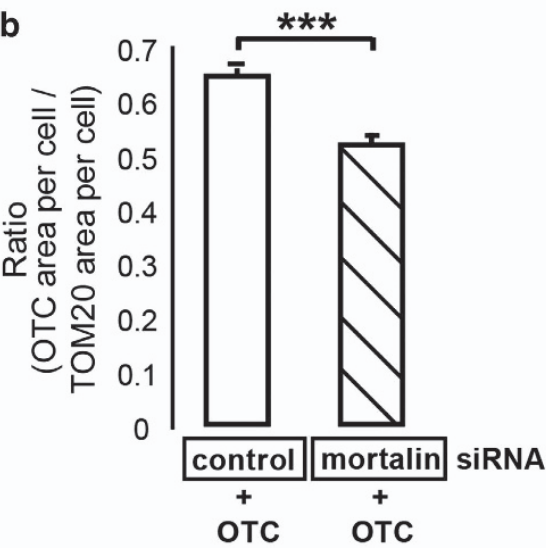

Figure 2 Intramitochondrial proteolytic stress phenocopies the reduced viability seen in loss of mortalin function models. (a) Intramitochondrial proteolytic stress in SH-SY5Y cells $(n=73)$ was genetically induced by overexpression of a truncated form of the matrix protein OTC referred to as dOTC. Immunocytochemical studies in fixed cells reveal both OTC and dOTC protein (GFP-green) located within mitochondrial structures shown as Tom20 signal (far red). siRNA transfection was confirmed by fluorescence of the Alexa555 siRNA tag (red). The expressed OTC protein is equally distributed in control cells, but is punctate in mortalin siRNA-treated cells. Quality control experiments to ensure equal expression of OTC and dOTC were performed (data not shown). Scale bars indicate $10 \mu \mathrm{m}$. (b) Subsequent ImageJ analysis was done from immunocytochemical studies in Figure 1a. The total area of mitochondria (Tom20 signal) colocalizing with OTC was less in the mortalin knockdown condition compared with the control condition

in the latter condition. This may indicate that the altered mitochondrial morphology readout reaches saturation, although we cannot rule out the possibility that less dOTC is imported into the mitochondria in the mortalin knockdown situation or that the level of proteolytic stress is saturated under two equally toxic conditions.
As fragmentation precedes the degradation of dysfunctional mitochondria, we investigated an additional downstream readout and measured whether the observed increased mitochondrial fragmentation translates into changes of mitochondrial mass. Knockdown of mortalin in SH-SY5Y cells reduced the abundance of the outer 

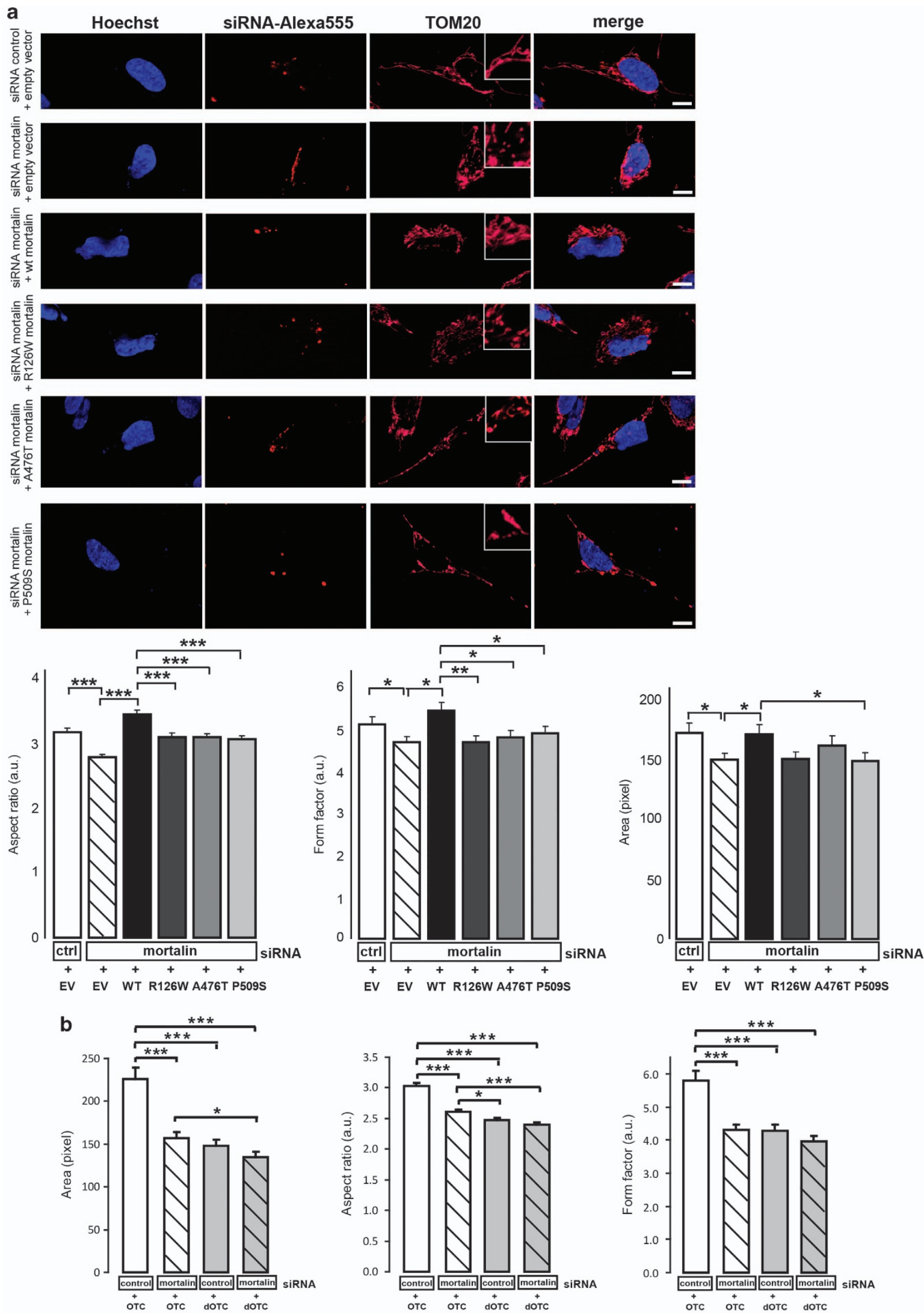

Figure 3 (Continued) 

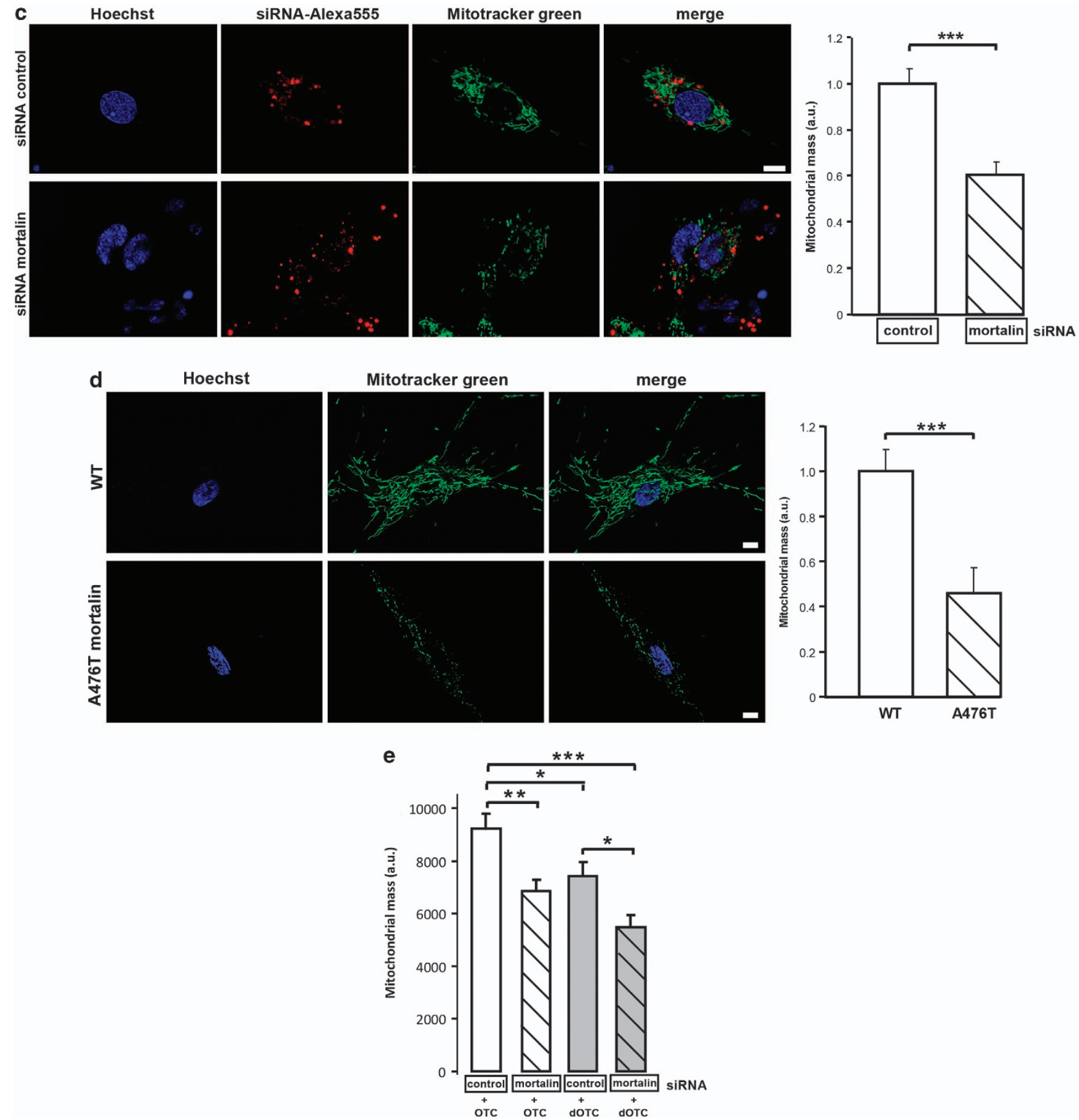

Figure 3 Proteolytic stress alters mitochondrial morphology in loss of mortalin function models. (a) Fluorescence microscopy in fixed cells ( $n=220$ cells) was used to analyze mitochondrial morphology in mortalin and control siRNA-treated SH-SY5Y with or without overexpressed wt mortalin or mortalin variants. Successful siRNA transfection was confirmed by fluorescence of the Alexa555 tag of the siRNA (red). Mitochondria were visualized by Tom20 antibody (far red) and Hoechst 33342 -stained nuclei (blue). Mortalin siRNA-treated cells have a smaller mitochondrial form factor, aspect ratio and mitochondrial size (area per mitochondrion). The reintroduction of wt mortalin but not the variants rescued this mitochondrial fragmentation phenotype. Ctrl, control; EV, empty vector. Scale bar indicates $10 \mu$ m. (b) Immunocytochemical studies in fixed SH-SY5Y cells $(n=138)$ treated with control siRNA or mortalin siRNA were additionally transfected with OTC or dOTC protein, and mitochondrial morphology analyzed. The expression of the dOTC protein leads to a fragmentation phenotype in control siRNA-treated cells. The aspect ratio as well as the form factor was decreased compared with control cells expressing the physiological OTC protein. The expression of OTC in a mortalin knockdown background phenocopied the control condition expressing dOTC. Mortalin knockdown cells with transiently transfected dOTC lead to the most severe mitochondrial fragmentation phenotype. (c) The mitochondrial mass of SH-SY5Y cells $(n=92)$ treated with mortalin siRNA or control siRNA (red) was analyzed by evaluating the total area of mitochondria (green) per cell via live cell imaging. Nuclei were visualized by DAPI stain (blue). Knockdown of mortalin caused a significant reduction in mitochondrial mass. Scale bar indicates $10 \mu \mathrm{m}$. (d) In human fibroblasts $(n=82)$, a reduced mitochondrial mass (green) was calculated for cells from a carrier of the A476T mortalin variant compared with controls via live cell imaging technique. Nuclei were visualized by DAPI stain (blue). Scale bar indicates $10 \mu \mathrm{m}$. (e) Induction of intramitochondrial proteolytic stress by expression of dOTC protein within siRNA control-treated cells leads to reduced mitochondrial mass measured by immunocytochemical studies in fixed cells and subsequent ImageJ analysis. Expression of OTC protein in mortalin knockdown cells caused a similar reduction of mitochondrial mass seen in control cells expressing dOTC protein. Expression of dOTC in mortalin siRNA-treated caused the most severe loss of mitochondrial structures. $N=138$ cells 
mitochondrial membrane protein Tom20 (Supplementary Figure $\mathrm{S} 1 \mathrm{~b}$ ) and the total area of mitochondria per cell (Figure $3 \mathrm{c}$ ), suggesting a reduction in mitochondrial mass. This was supported by live cell imaging analyses in A476Tfibroblasts stained with Mitotracker green, a mitochondrial dye not dependent on MMP (Figure 3d). In line with intramitochondrial proteolytic stress modulating mitochondrial dynamics, the expression of dOTC within control siRNAtreated cells led to a significant reduction of mitochondrial mass (Figure 3e). Again, combining mortalin knockdown and expression of dOTC resulted in a more severe reduction of mitochondrial mass. This indicates synergistic intramitochondrial proteolytic stress conditions in terms of overall mitochondrial mass.

Knockdown of mortalin elevates autophagic activity and the specific degradation of mitochondria. Because of compensatory induction of organellar quality control mechanisms, intramitochondrial proteolytic stress should promote the specific degradation of dysfunctional mitochondria through autophagic clearance. To address this possibility, we employed several measures to assess autophagy by quantitative fluorescence microscopy techniques: (1) assessment of overexpressed GFP-LC3 puncta by confocal microscopy; (2) endogenous WIPI-2 puncta formation by confocal microscopy; (3) automated high-throughput imaging and analysis of endogenous p62; and (4) colocalization of lysosomes and mitochondria. Indeed, we found that mortalin knockdown results in a significantly higher level of autophagic activity and mitochondrial-lysosomal colocalisation, a measure of mitophagy.

In detail, we used transiently overexpressed GFP-LC3 in SH-SY5Y to assess the number of autophagosomes by quantitative fluorescence microscopy. ${ }^{32}$ We found that the number of GFP-LC3 puncta per cell significantly increased in response to mortalin knockdown (Figure 4a), and this was abolished by overexpressing wt mortalin, but only partially by any of the PD-associated mortalin variants (Figure 4a). An increased number of autophagosomes (GFP-LC3 puncta) per cell can either be the result of an increased level in autophagy initiation or of a block in later stages of autophagy when autophagosomes contact the lysosomal compartment for final cargo degradation (autophagic flux). ${ }^{33}$ Hence, we monitored the autophagic flux by standard cotreatments with the lysosomal inhibitor bafilomycin A1 (BafA1) that prevents the fusion between autophagosomes and lysosomes. As expected, the addition of BafA1 elevated the number of GFP. LC3 puncta per cell under control conditions (Figure 4b). The number of GFP-LC3 puncta per cell clearly increased upon BafA1-treated mortalin knockdown when compared with both untreated mortalin knockdown cells and control siRNA cells treated with BafA1 (Figure 4b). This result strongly indicates that the autophagic activity increases upon mortalin knockdown.

For further autophagy assessments we employed SH-SY5Y cells with inducible expression of miRNA targeting mortalin (Figure 4c and Supplementary Figures S2-S4). By analyzing endogenous LC3 lipidation, the conjugation of LC3 (LC3-I) to phosphatidylethanolamine (LC3-II), we confirmed proper autophagic activity in this cellular model
(Supplementary Figure S2). Next, we monitored the specific localization of endogenous WIPI-2, a member of the WIPI protein family ${ }^{34}$ that functions as an essential phosphotidylinositol-3-phosphate (Ptdlns(3)P) effector during autophagy initiation. ${ }^{35}$ Upon autophagy initiation, WIPI-2 accumulates at early and late autophagosomal membranes, ${ }^{36,37}$ and hence autophagy initiation can also be assessed by quantitative fluorescent puncta formation. In line with our results using GFP-LC3, we found a significant increase of endogenous WIPI-2 puncta when mortalin is knocked down (Figure 4c and Supplementary Figure S3). Furthermore, using an automated high-throughput imaging and analysis platform, ${ }^{37}$ we analyzed the specific targeting of endogenous p62 by autophagic clearance and found an increase in p62 degradation in cells lacking endogenous mortalin (Supplementary Figure S4). In summary, our autophagy assessments strongly indicate that mortalin negatively controls the process of autophagy and that mortalin knockdown results in elevated autophagic activity.

As autophagy controls both the function and numbers of cellular organelles including mitochondria, we addressed the question of whether or not autophagy activation upon mortalin knockdown would lead to an elevated level of specific mitochondrial clearance called mitophagy (Figure 4d). For this aim, we quantified the colocalization of mitochondria, marked with Tom20 antibodies, with lysosomes, marked with LAMP1 antibodies, as a measure of mitophagy. ${ }^{33}$ Indeed, we found a higher degree of colocalization in cells treated with mortalin siRNA compared with controls, indicating increased autophagic clearance of dysfunctional mitochondria (Figure 4d). This effect was abolished by wt mortalin overexpression into the knockdown background but not by mutant R126W or P509S mortalin variants (Figure 4d). Although mutant A476T mortalin was able to revert the phenotype of acute mortalin knockdown in human SH-SY5Y cells, the chronic ex vivo model of human A476T fibroblasts revealed an increased mitochondrial-lysosomal colocalization compared with controls, and therefore supports a concept of compensatory upregulation of organellar quality control. ${ }^{23}$

Finally, we investigated the effect of mortalin knockdown in mouse embryonic fibroblasts (MEFs) from ATG5 knockout (KO) mice that contain an inducible ATG5 cDNA under the control of doxycycline. ${ }^{38}$ ATG5 is an essential component of autophagy-specific conjugation systems that promote the conjugation of LC3 to phosphatidylethanolamine at the forming autophagosome, and hence ATG5 deficiency blocks autophagy at early stages. In the presence of ATG5, a significant increase in the number of GFP-LC3 puncta per cell upon knockdown of mortalin was found (Figure 4e), corresponding to our findings using the neuronal cell line SH-SY5Y (Figures $4 \mathrm{a}-\mathrm{c}$ and Supplementary Figures S3 and S4). In contrast, in MEFs devoid of ATG5 the increase of GFP-LC3 puncta upon mortalin knockdown was completely abolished. This indicates that mortalin controls the canonical autophagic machinery. ${ }^{35}$

Loss of mortalin function leads to enhanced activation of caspase 3 and apoptotic cell death. To investigate the potential relevance of changes of mitochondrial morphology and induction of autophagy after loss of mortalin function to cell death, we analyzed cellular viability by measuring the 
activation of caspase 3 and the appearance of apoptotic nuclei. Acute mortalin depletion was sufficient to induce apoptotic cell death in vitro (Figure 5a).

To further challenge molecular quality control in the mitochondria, we chemically induced intramitochondrial stress via 17-allylamino-17-demethoxygeldanamycin (17-AAG) treatment of cells. Previously, 17-AAG was described as an inhibitor of the chaperone Hsp90 in cytoplasm and mitochondria, thereby eliciting a $\operatorname{UPR}(\mathrm{mt})$ that promotes apoptosis. ${ }^{39,40}$ Knockdown of mortalin sensitized SH-SY5Y cells to 17-AAG treatment as quantified by the percentage of apoptotic nuclei and the activation of caspase 3 (Figure $5 b$ ). It is noteworthy that overexpression of wt mortalin in control SH-SY5Y cells treated with 17-AAG apparently compensated for the reduced $\mathrm{Hsp} 90$ activity and protected against subsequent apoptosis. In addition, in human A476T-fibroblasts we observed an increased susceptibility to 17-AAG treatment with higher levels of apoptosis compared with control cells (Figure 5c). To investigate the specificity of the loss of mortalin function-mediated susceptibility toward increased mitochondrial proteolytic stress, we treated cells with thapsigargin (TG), an inducer of endoplasmic reticulum (ER) stress. ${ }^{41}$ ER-stress caused apoptosis to a similar extent in both control and mutant fibroblasts (Figure $5 \mathrm{~d}$ ).

The application of more specific genetically induced intramitochondrial proteolytic stress by overexpression of the truncated dOTC protein increased apoptosis in control SH-SY5Y cells to a similar extent as the chemical treatment (Figure 5e). Increased apoptotic nuclei but not activation of caspase-3 by dOTC was further exacerbated in the mortalin knockdown situation, suggesting that the apoptotic mechanism promoted by mortalin-induced proteolytic stress does not require caspase- 3 and can act via alternative execution pathways. Notably, the overexpression of native OTC in a mortalin knockdown background induced apoptosis to a similar extent as control cells transfected with dOTC. This indicates that proteolytic stress due to dOTC overexpression recapitulates the effect of mortalin knockdown in terms of cellular viability. Knockdown of mortalin in combination with the expression of the dOTC protein showed the most severe effect on cell viability.
Increased organellar quality control due to overexpression of either PINK1 or Parkin rescues loss of mortalin function phenotypes. The enhanced mitophagy observed in response to mortalin knockdown might exacerbate defects because of a depletion of mitochondria. Alternatively, it might be essential to prevent further cellular damage. To address this question, we sought to modulate autophagy in cells affected by the loss of mortalin function.

Chemical induction of autophagy by Rapamycin reduced apoptosis in mortalin knockdown models as revealed by decreased caspase 3 activation and a reduced number of apoptotic nuclei (Figure 6a), as well as a reduced Annexin V staining revealed by FACS analysis (Supplementary Figure S5). In line with these observations, an inhibition of autophagy by use of the lysosomal inhibitor BafA1 in mortalin knockdown cells exacerbated apoptosis (Figure 6b). This clearly supports a critical role of functional autophagic machinery to prevent further damage.

Intriguingly, the mitochondrial quality control machinery is critically related to PD genes encoding PINK1 and Parkin as mediators of the autophagic degradation of dysfunctional mitochondria. Therefore, to reduce the proportion of nonfunctional mitochondria among the mitochondrial pool, we genetically upregulated mitochondrial quality control proteins $^{28}$ by overexpressing either PINK1 or Parkin in cells treated with mortalin siRNA and monitored the resulting phenotypes. Both PINK1 and Parkin overexpression in a mortalin knockdown background resulted in significantly enhanced numbers of GFP-LC3 puncta compared with the control condition (Figure 6c). These results argue in favor of an activation of autophagy to degrade impaired mitochondria. Consistently, the mitochondrial fragmentation phenotype in mortalin knockdown cells was rescued by overexpression of either PINK1 or Parkin (Figure 6d).

To define whether our observations on positive effects of PINK1 and Parkin on mitochondrial network homeostasis were related to increased clearance of fragmented mitochondria from the cell, we used the ATG5 KO model in MEFs. In control MEFs expressing ATG5, PINK1 partially and Parkin fully rescued the mitochondrial phenotype upon mortalin knockdown, in line with our observations in human neuronal

Figure 4 Proteolytic stress causes increased mitochondrial clearance loss of mortalin function models. (a, left panel) Fluorescence microscopy in fixed cells ( $n=252$ cells) was used to analyze the number of autophagosome by overexpressing GFP-LC3 (green) in mortalin and control siRNA-treated SH-SY5Y. Cells were transfected with wt mortalin or mortalin variants in the ratio of 1:4 together with GFP-LC3 (controls for positive transfection of the mortalin plasmid). Successful siRNA transfection was confirmed by fluorescence of the Alexa555 tag of the siRNA (red). Hoechst 33342 stained nuclei (blue). (a, right panel) Mortalin siRNA-treated cells have a higher number of GFP-LC3 puncta compared with control siRNA-treated cells. The reintroduction of wt mortalin but not the variants rescued this phenotype. Scale bar indicates $10 \mu \mathrm{m}$. (b, left panel) Use of the autophagy inhibitor Bafilomycin A1 (BafA1) in SH-SY5Y cells $(n=188)$ leads to enhanced numbers of GFP-LC3 puncta (green) in both control and mortalin siRNA-treated cells (red). (b, right panel) Mortalin siRNA-treated cells have a higher number of GFP-LC3 puncta compared with control siRNA-treated cells. Treatment with BafA1 resulted in a further increase of GFP-LC3 puncta in the mortalin knockdown as well as control condition. Scale bar indicates $10 \mu \mathrm{m}$. (c) SH-SY5Y cells expressing mortalin miRNA or control miRNA for 24, 48 or $72 \mathrm{~h}$ were treated with EBSS and Bafilomycin A1 (BafA1) followed by anti-WIPI-2 immunoflourescence. Confocal fluorescence microscopy (Zeiss LSM 510) image projections ( $n=10$ ) were used (representative images in the left panels) to assess the number of endogenous WIPI-2 puncta per cell using ImageJ software analysis (right panels). Knockdown of mortalin (black bars) significantly increased the number of WIPI-2 puncta per cell (or the total fluorescent area; Supplementary Figure S3) when compared with control cells (gray bars). Scale bar indicates $20 \mu \mathrm{m}$. (d) Fluorescence microscopy in fixed cells ( $n=244$ cells) was used to analyze mitochondrial-lysosomal colocalization in mortalin and control siRNA-treated SH-SY5Y with or without overexpressed wt mortalin or mortalin variants. Successful siRNA transfection was confirmed by fluorescence of the Alexa555 tag of the siRNA (red). Mitochondria were visualized by Tom20 antibody (far red), lysosomes by Lamp-1 (green) and Hoechst 33342-stained nuclei (blue). Mortalin siRNA-treated cells revealed a higher value for the Pearson coefficient as a measure for colocalization rate. The reintroduction of wt mortalin but not the R162W or P509S mortalin variants rescued this phenotype. A476T mortalin showed no significant difference to the wt mortalin condition. Ctrl, control; EV, empty vector. Scale bar indicates $10 \mu \mathrm{m}$. (e) Knockdown of mortalin in ATG5WT MEFs along with transient expression of GFP-LC3 resulted in an increased number of autophagosomes (GFP-LC3 puncta) compared with control siRNA-treated ATG5 WT MEFs ( $n=72$ cells). In ATG5 KO MEFs ( $n=82$ cells), transient expression of GFP-LC3 did not result in an increased puncta formation, because the formation of autophagosomes was abolished. It is noteworthy that the absolute number of GFP-LC3 puncta may differ between mortalin knockdown in human versus murine cells, as expected. ${ }^{33} \mathrm{Ctrl}$, control 
SH-SY5Y cells (Figure 7a). In contrast, neither PINK1 nor Parkin overexpression rescued the mitochondrial fragmentation in ATG5 KO MEFs (Figure 7b). The wt mortalin overexpression resulted in a healthy mitochondrial network in both ATG5 WT and KO MEFs. This may indicate that the mechanism of rescue by overexpression of wt mortalin is not dependent on autophagosome formation (organellar quality control), but may reduce the intramitochondrial proteolytic disturbances resulting from mortalin knockdown. Whereas the rescue by wt mortalin overexpression acts within the mitochondria, an overexpression of either PINK1 or Parkin acts at the autophagy stage and cannot be successful in cells devoid of autophagosomes.

To define whether an increased mitochondrial clearance in mortalin knockdown cells may be beneficial or rather toxic for the cells, we measured apoptotic cell death by Annexin V analysis. We found reduced levels of apoptosis under conditions of induced autophagy after overexpression of Parkin in mortalin knockdown cells (Figure 7c). This argues for a role of Parkin in rescuing the loss of mortalin by induction of
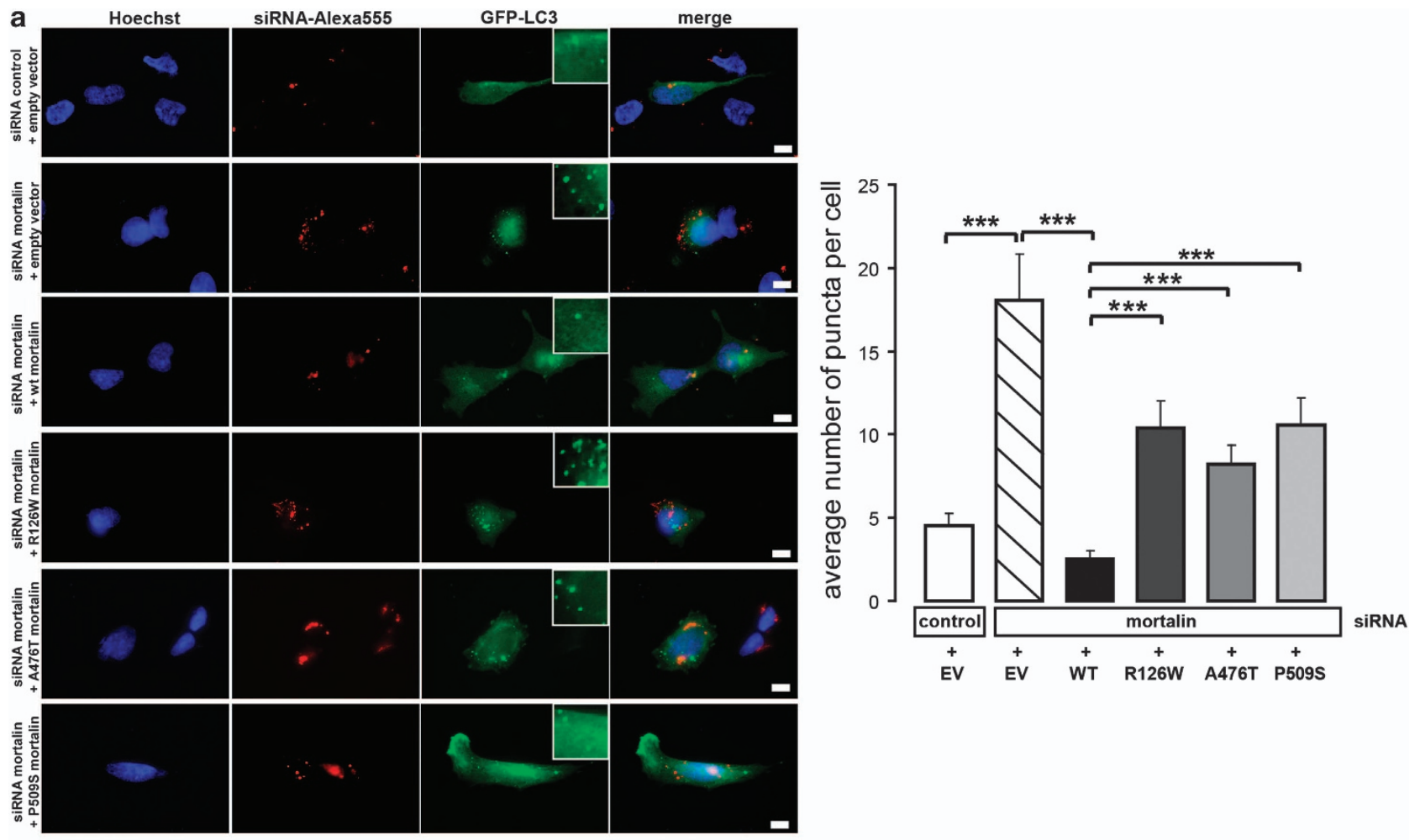

b
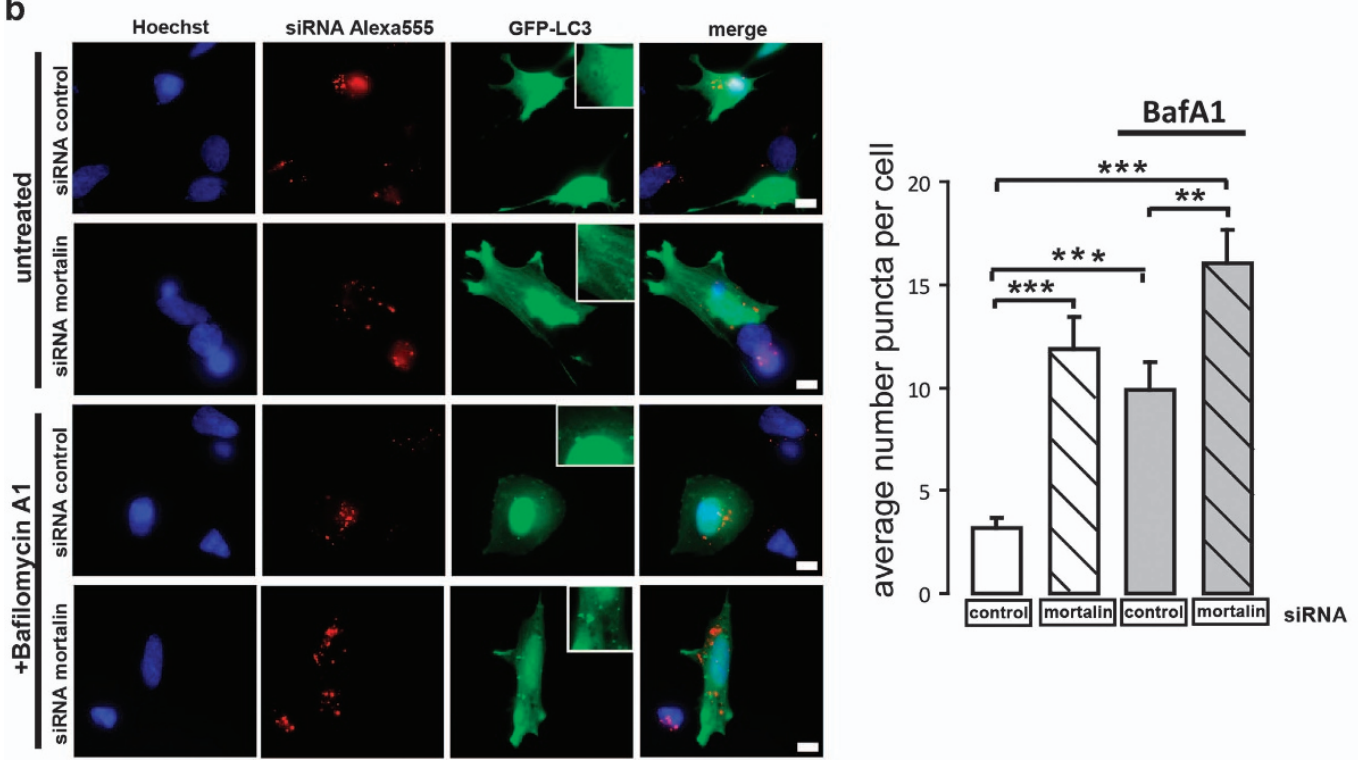

Figure 4 (Continued) 
autophagy in mortalin siRNA-treated cells (Figure 7c). PINK1 overexpression showed an effect on cell survival, but was not statistically significant. Finally, we investigated the link between mortalin loss of function and Parkin-mediated mitophagy by assessing the effect of expressing myc-Parkin in our mortalin knockdown paradigm. Therefore, we assessed an early stage of Parkin-mediated mitophagy that is defined by clustering of mitochondria and that precedes mitochondrial
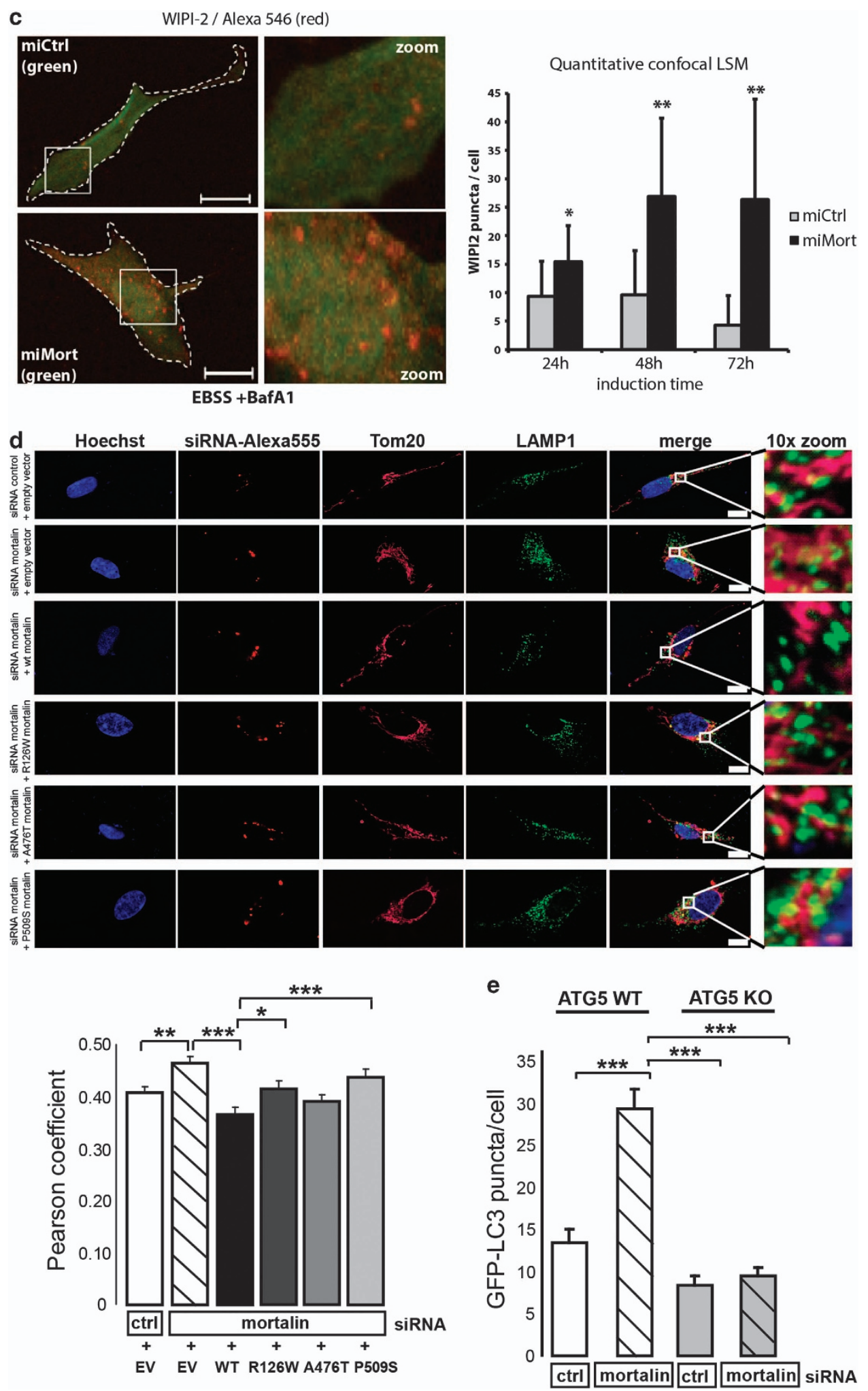

Figure 4 (Continued) 
removal. ${ }^{42}$ We knocked down mortalin using an inducible miRNA system in HeLa cells, which are devoid of endogenous Parkin, ${ }^{42}$ and introduced recombinant myc-Parkin in mortalin knockdown as well as control cells. In accordance with our previous finding in $\mathrm{SH}-\mathrm{SY} 5 \mathrm{Y}$ cells and patient fibroblasts, the mitochondria in HeLa cells were fragmented when mortalin is knocked down. However, a significant increase in mitochondrial clustering as a readout for the induction of mitophagy was only observed after the introduction of Parkin in mortalin knockdown cells (Figure $7 d$ ). These sequestered

\section{a}
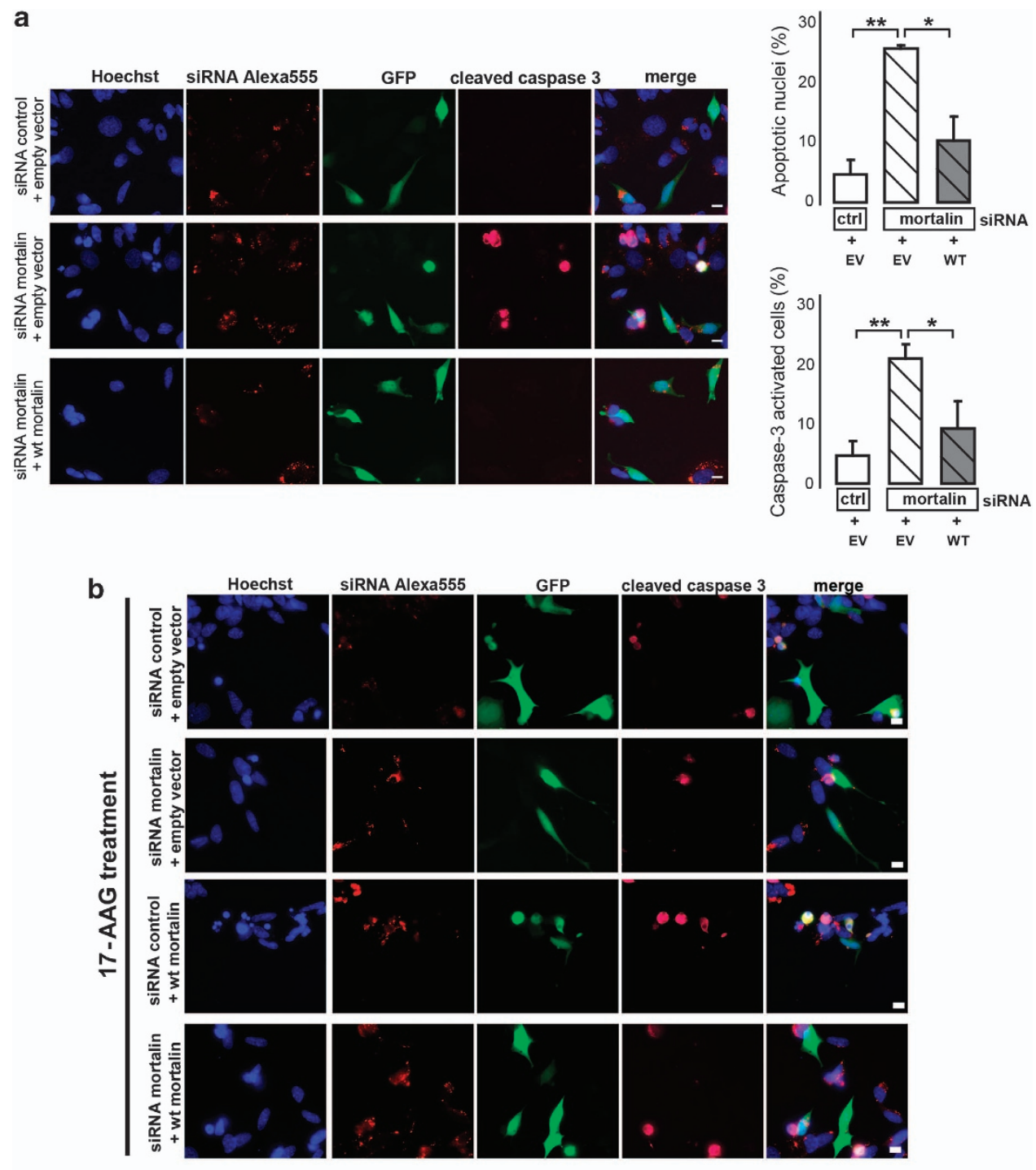

cleaved caspase
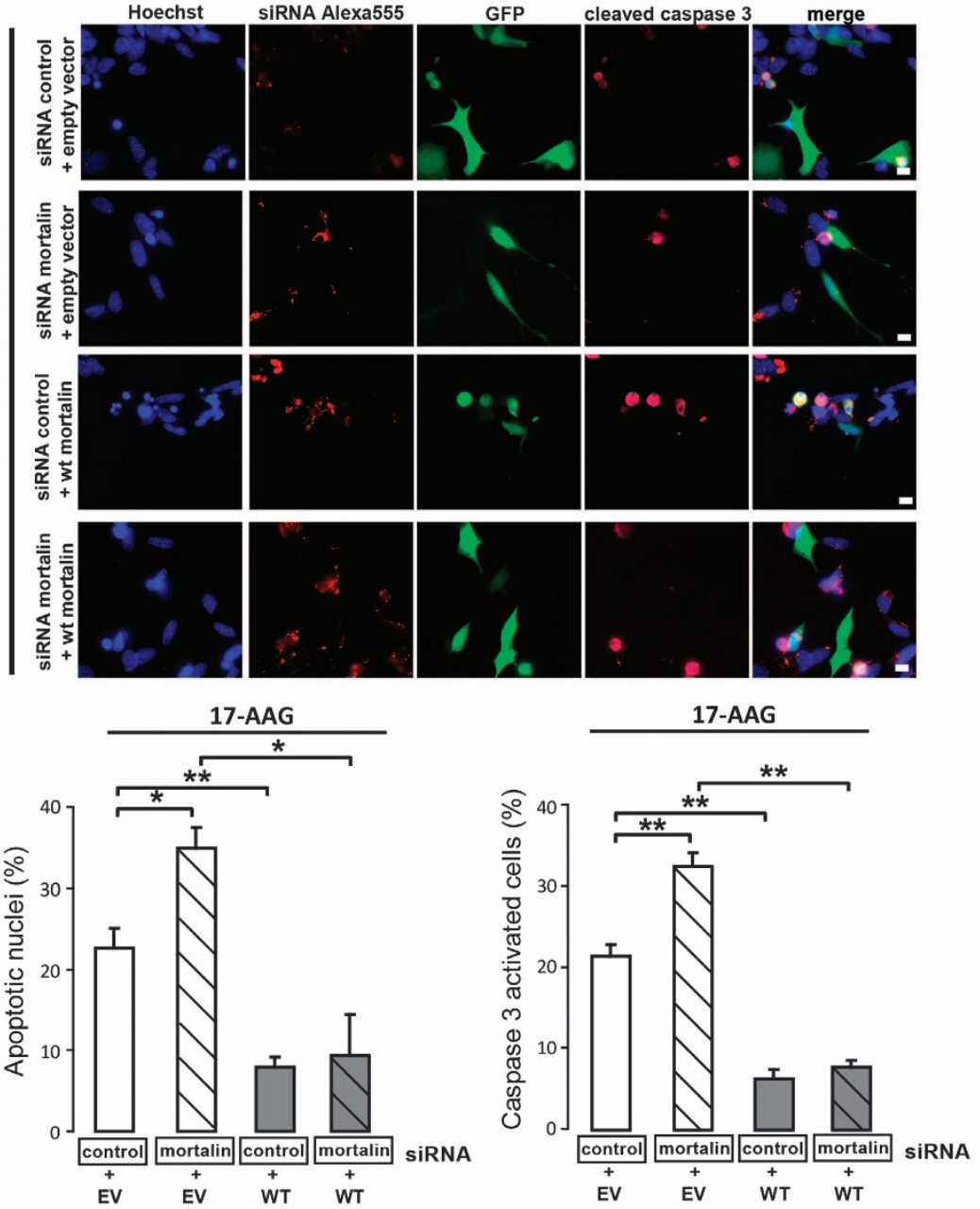

Figure 5 (Continued) 

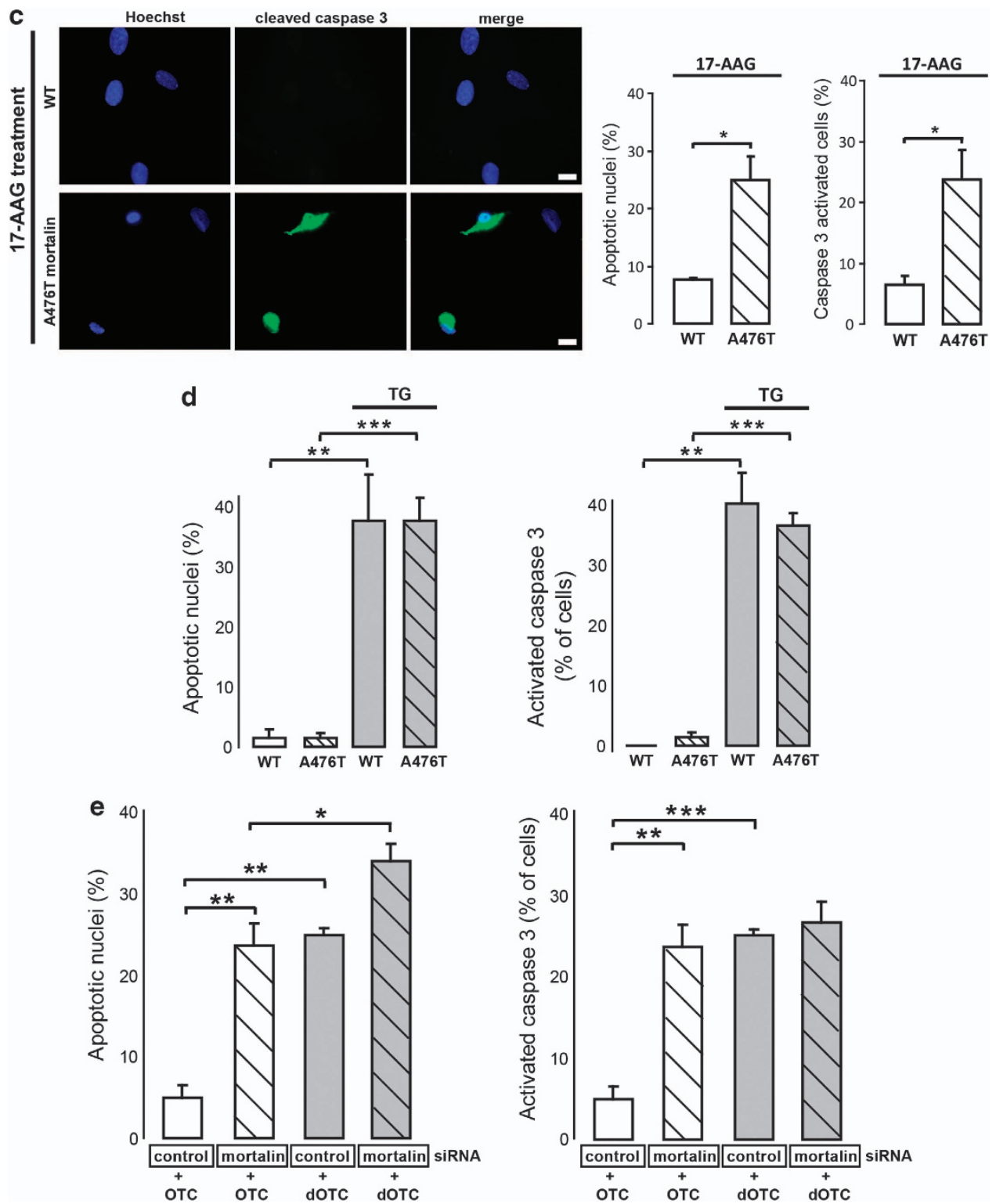

Figure 5 Impaired mitochondrial proteostasis leads to increased apoptosis in human cells with reduced mortalin levels and is rescued by induction of the autophagic pathway (a, left panel) Fluorescence microscopy in fixed cells $(n=272$ cells) was used to analyze the number of apoptotic nuclei by Hoechst staining (blue) and a positive signal for activated caspase 3 (far red). Cells were transfected with wt mortalin or empty vector in the ratio of 1:4 together with an empty eGFP-N1 vector (controls for positive transfection of the mortalin plasmid). Successful siRNA transfection was confirmed by fluorescence of the Alexa555 tag of the siRNA (red). (a, right panel) Mortalin siRNAtreated cells have a higher number of apoptotic nuclei and cells with activated caspases 3 . The reintroduction of wt mortalin rescued this apoptotic cell death phenotype. Ctrl, control; EV, empty vector. Scale bar indicates $10 \mu \mathrm{m}$. (b) The pharmacological compound 17-AAG was used for induction of intramitochondrial proteolytic stress in fixed SHSY5Y cells. Cells were transfected with wt mortalin or empty vector in the ratio of $1: 4$ together with an empty eGFP-N1 vector (controls for positive transfection of the mortalin plasmid). Successful siRNA transfection was confirmed by fluorescence of the Alexa555 tag of the siRNA (red). Treatment with 17-AAG in mortalin siRNA-treated cells leads to enhanced numbers of apoptotic nuclei (blue) and activation of caspase 3 (far red). Overexpression of wt mortalin fully reversed the phenotype in both control and mortalin knockdown cells. Scale bar indicates $10 \mu \mathrm{m}$. (c) Fluorescence microscopy in fixed human fibroblasts $(n=206)$ revealed enhanced apoptosis in terms of an increase in apoptotic nuclei (blue) and in caspase 3 activation (green) in cells from a carrier of the A476T mortalin variant compared with control fibroblasts upon induction of intramitochondrial proteolytic stress via 17-AAG treatment. Scale bar indicates $10 \mu \mathrm{m}$. (d) Induced specific ER stress by treatment with thapsigargin (TG) causes apoptosis to a similar extent in control as well as in fibroblasts from a carrier of the A476T mortalin variant $(n=336)$. Induction of ER stress increased the amount of apoptotic nuclei as well as caspase-3 activation in control and mutant fibroblast to the same amount ( $>35 \%$ ), underscoring the specificity of intramitochondrial stress due to loss of mortalin function. (e) Expression of the dOTC protein leads, in both control and mortalin knockdown SH-SY5Y cells $(n=428)$, to an increase in apoptotic nuclei as well as in caspase 3 activation compared with the respective counterparts with expression of the OTC protein. Transfection of the OTC protein in mortalin siRNA-treated cells caused similar amount of apoptosis compared with the condition of dOTC expression in control cells. The increase in apoptotic nuclei but not in the activation of caspase-3 upon expression of dOTC in the mortalin knockdown situation suggests that the apoptotic mechanism promoted by mortalin-induced proteolytic stress does not require caspase-3 and can act via alternative execution pathways 

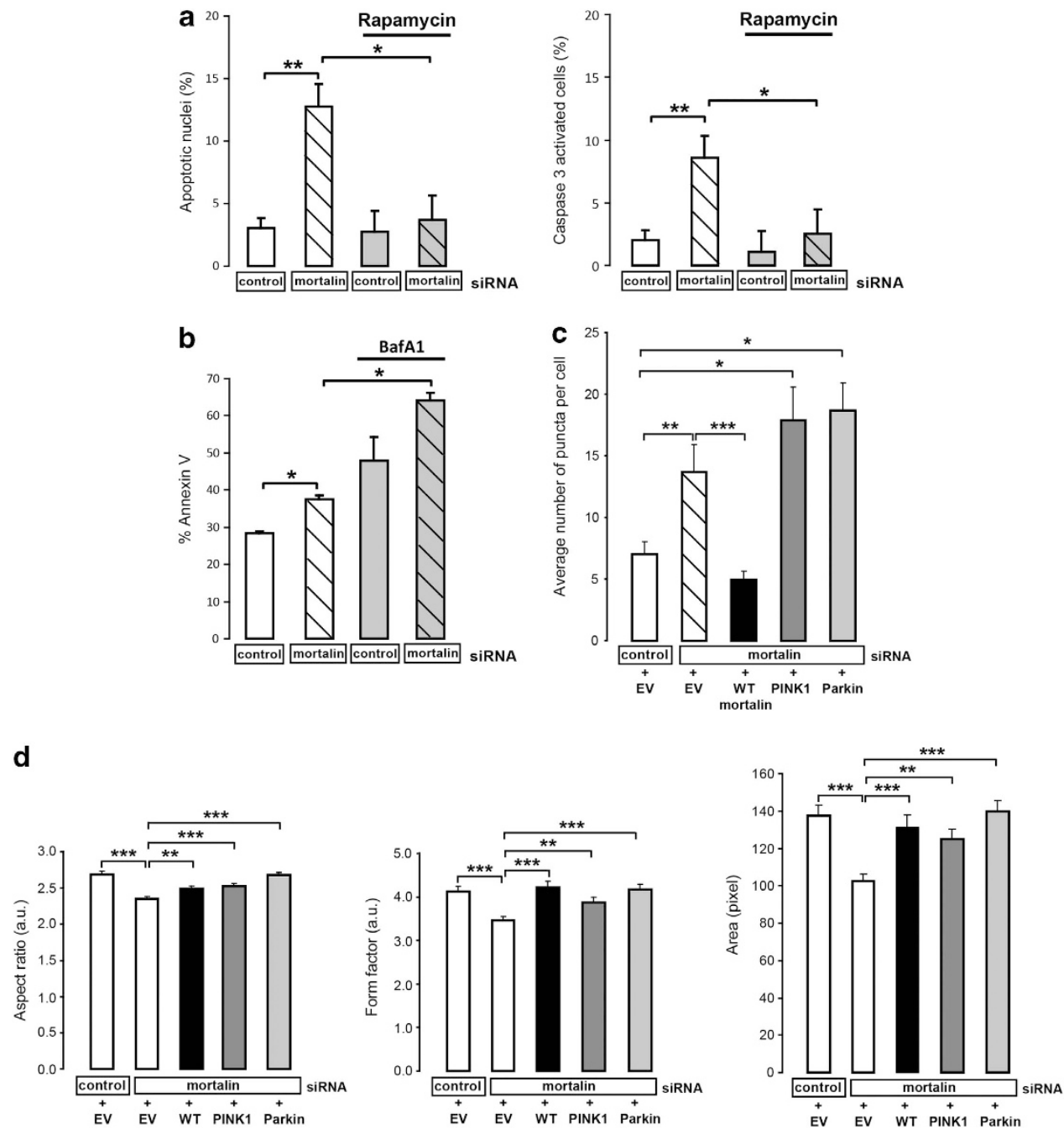

Figure 6 Rapamycin treatment or overexpression of PINK1 or Parkin rescues cellular phenotypes associated with loss of mortalin function (a) Cells were treated with control siRNA or mortalin siRNA and analyzed for apoptotic nuclei and activation of caspase 3 via immunofluorescence studies. The induction of autophagy by treatment with Rapamycin resulted in a significantly lower amount of caspase 3 activation as well as apoptotic nuclei in the mortalin knockdown condition compared with the control condition. (b) Cells were treated with control siRNA or mortalin siRNA and analyzed for apoptotic cell death via Annexin V staining measured by FACS analysis. Knockdown of mortalin resulted in an increased Annexin V signal. The inhibition of lysosomal function by treatment with BafA1 enhanced the Annexin V signal in both control and mortalin knockdown condition. (c) Fluorescence microscopy in fixed cells ( $n=197$ cells) was used to analyze the number of autophagosome by overexpressing GFP-LC3 (green) in mortalin and control siRNA-treated SH-SY5Y. Cells were transfected with wt mortalin, PINK1 or Parkin in the ratio of 1:4 together with GFP-LC3 (controls for positive transfection of the mortalin plasmid). Mortalin siRNA-treated cells have a higher number of GFP-LC3 puncta compared with control siRNA-treated cells. The overexpression of wt mortalin resulted in a decreased number of GFP-LC3 puncta, whereas the overexpression of PINK1 or Parkin was followed by an enhanced number of GFP-LC3 puncta compared with the control condition. (d) Fluorescence microscopy in fixed cells $(n=214$ cells) was used to analyze mitochondrial morphology in mortalin and control siRNA-treated SH-SY5Y with or without overexpressed wt mortalin, PINK1 or Parkin. Mortalin siRNA-treated cells have a smaller mitochondrial form factor, aspect ratio and mitochondrial size (area per mitochondrion). The reintroduction of either wt mortalin, PINK1 or Parkin rescued this mitochondrial fragmentation phenotype

mitochondria were previously described in paradigms of carbonyl cyanide $m$-chlorophenyl hydrazone (CCCP)-induced mitophagy. ${ }^{42,43}$ In sum, the functional quality control system, enhanced by the overexpression of PINK1 or Parkin, lead to the activation of autophagy and in consequence to a rescue of the mitochondrial network in mortalin knockdown cells, thereby explaining the ability of Parkin to rescue mortalin knockdown cells from apoptosis.

\section{Discussion}

The genetic basis for disrupted mitochondrial integrity as a cause of neurodegeneration in PD was uncovered by the functional characterization of mutations that cause loss of Parkin or PINK1 function in autosomal recessive forms of the disease. A linear signaling pathway, with PINK1 acting upstream of Parkin, was described. ${ }^{24,25}$ This pathway controls the proper clearance of dysfunctional mitochondria from the cell. $^{25}$ In this context, PINK1 is stabilized on the outer membrane of defective mitochondria and activates the process that leads to the recruitment of the E3 ubiquitin ligase Parkin to the dysfunctional organelle, finally leading to lysosomal degradation. ${ }^{44}$ Recently, the PINK1-mediated phosphorylation of mitofusin 2 (Mfn2) was found to be the critical step for the initiation of mitophagy via ubiquitination of Mfn2, with phosphorylated Mfn2 acting as a receptor for Parkin. ${ }^{45}$ 
a

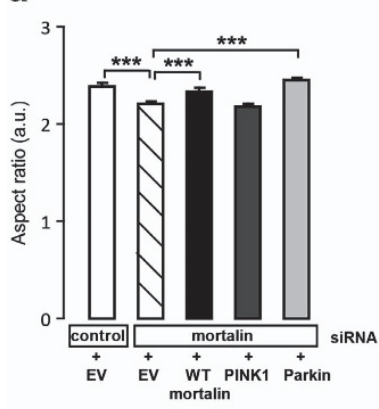

b

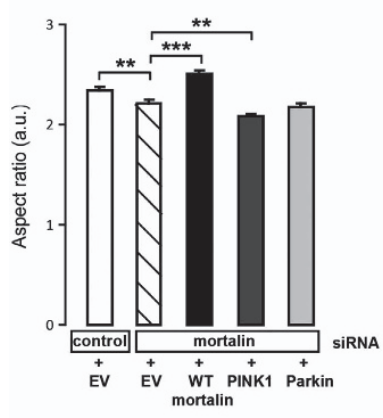

C

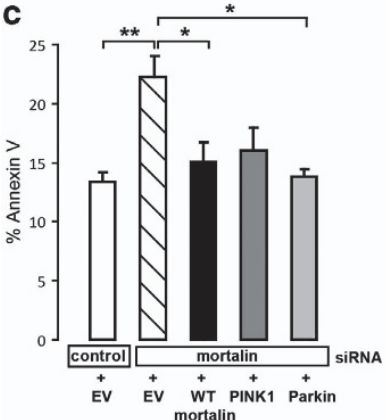

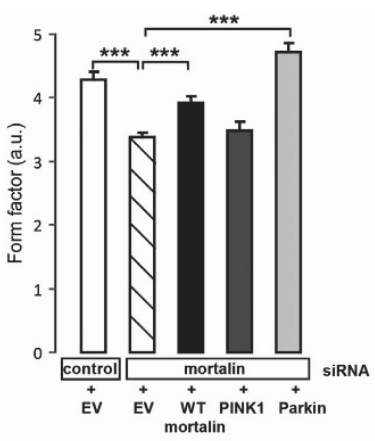
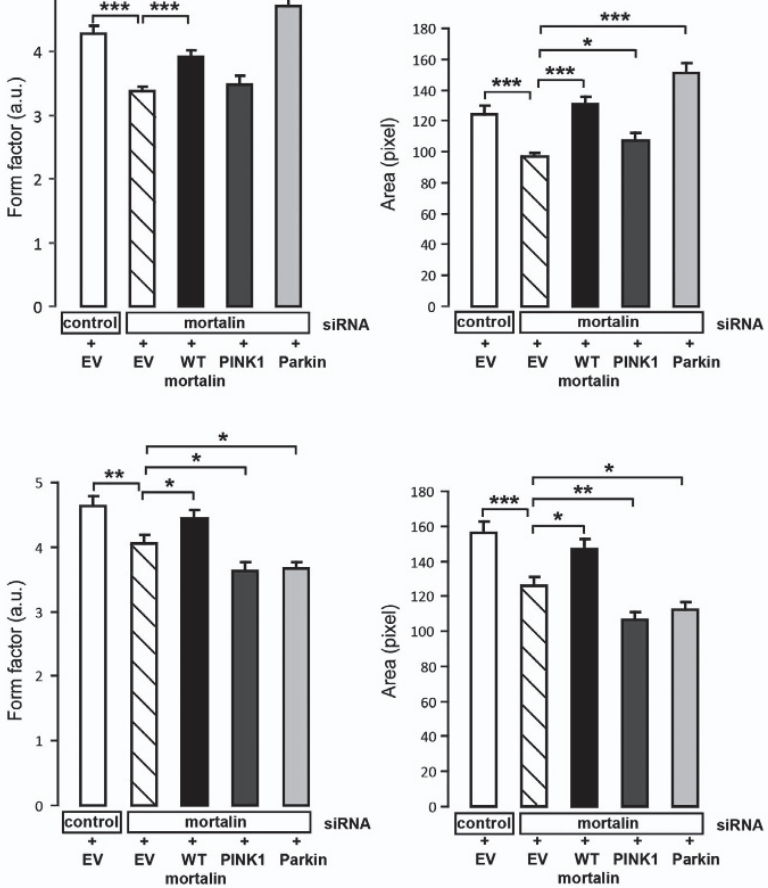

d
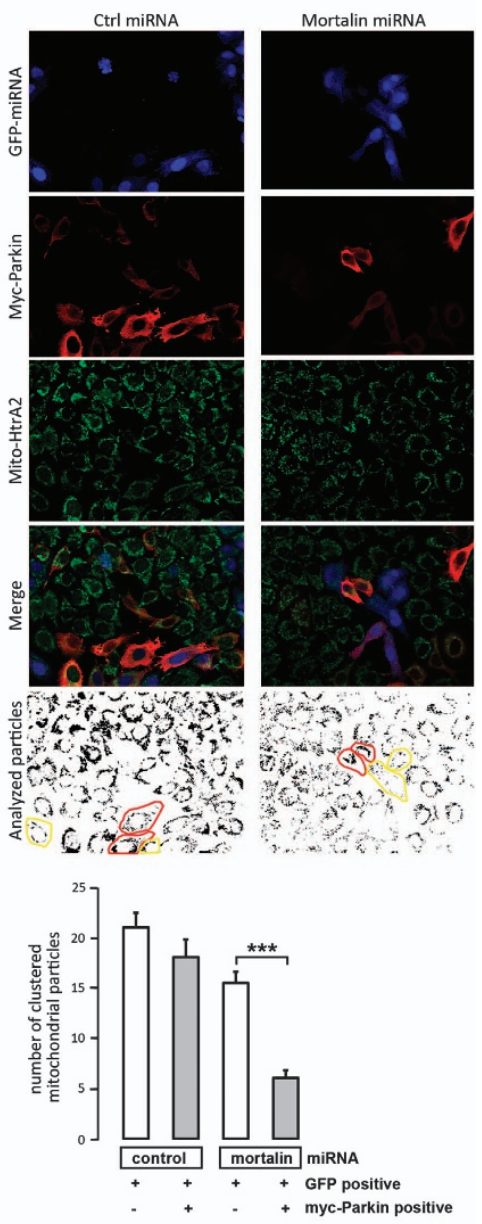

Figure 7 Rescue of mitochondrial fragmentation phenotype due to mortalin knockdown by Parkin overexpression requires an intact autophagic machinery leading to reduced cell death. (a) Fluorescence microscopy in fixed cells ( $n=94$ cells) was used to analyze mitochondrial morphology in mortalin and control siRNA-treated MEFs with or without overexpressed wt mortalin, PINK1 or Parkin. Mortalin siRNA-treated cells have a smaller mitochondrial form factor, aspect ratio and mitochondrial size (area per mitochondrion). The reintroduction of either wt mortalin or Parkin rescued this mitochondrial fragmentation phenotype. Reintroduction of PINK1 resulted only partially in the rescue of mitochondrial fragmentation. This suggests that successful rescue through Parkin overexpression is dependent on ATG5 (compare also Figure $7 \mathrm{~b}$ ), whereas PINK1 does not appear to be affected by ATG5. (b) Fluorescence microscopy in fixed cells ( $n=102$ cells) was used to analyze mitochondrial morphology in mortalin and control siRNA-treated ATG5 KO MEFs with or without overexpressed wt mortalin, PINK1 or Parkin. Mortalin siRNA-treated cells have a smaller mitochondrial form factor, aspect ratio and mitochondrial size (area per mitochondrion). Only the reintroduction of wt mortalin rescued this mitochondrial fragmentation phenotype, whereas neither overexpression of PINK1 nor of Parkin did. (c) SH-SY5Y cells were treated with control siRNA or mortalin siRNA and analyzed for apoptotic cell death via Annexin V staining measured by FACS analysis. Knockdown of mortalin resulted in an increased Annexin V signal. Overexpression of wt mortalin or Parkin in cells treated with mortalin siRNA led to a full rescue of this phenotype. PINK1 overexpression lowered the amount of Annexin V staining, but did not significantly rescue the observed phenotype in mortalin knockdown cells. (d) Knockdown of mortalin in HeLa cells was achieved by inducible miRNA system. Myc-Parkin was transiently transfected. The clustering of mitochondria was assessed by counting the number of mitochondrial particles per cell in those with a positive signal for miRNA only or for miRNA + myc-Parkin in knockdown mortalin cells as well as control cells. Knockdown of mortalin resulted in fragmentation of the mitochondrial network, whereas the exogenous introduction of myc-Parkin led to mitochondrial clustering around the nuclear region. Cells overexpressing myc-Parkin are encircled in red. Cells overexpressing without exogenous expression of myc-Parkin are encircled in yellow

Our results now provide evidence for an involvement of impaired intramitochondrial molecular quality control in PD mediated by loss of mortalin function. Importantly, mitochondrial dysfunction and altered integrity were compensated by PINK1/Parkin-mediated autophagy, representing downstream organellar quality control. We found that loss of mortalin causes intramitochondrial proteolytic stress in vitro and ex vivo as defined by increased expression level of
Hsp60, a marker for the activation of the UPR $(\mathrm{mt}){ }^{29}$ Activation of the UPR $(\mathrm{mt})$ was further supported by the observed mitonuclear protein imbalance and an accumulation of insoluble proteins within the mitochondria. Consistently, human neuronal cell lines with reduced levels of mortalin were more vulnerable to chemically or genetically induced intramitochondrial proteolytic stress, an effect that was also observed in fibroblasts from a carrier of the A476T variant 
and that was completely rescued by reintroduction of wt mortalin. In contrast, no rescue capacity for mortalin was observed for pharmacologically induced ER-stress in this model, indicating the specificity of mortalin for $\operatorname{UPR}(\mathrm{mt})$ and the importance of mortalin for the maintenance of proteostasis within the mitochondrial matrix. Thus, we propose a linear signaling cascade in which loss of mortalin triggers impaired mitochondrial proteostasis that is upstream of PD-related neurodegeneration (Figure 8).

In our experiments in different in vitro and ex vivo models of loss of mortalin function, we found evidence for increased autophagy due to accumulating mitochondrial damage based on intramitochondrial proteolytic stress. We demonstrate that the accumulation of autophagosomes upon loss of mortalin is because of activated autophagy. Although the correlation between the effect on autophagic activation and mitochondrial clearance is difficult to assess, the consistent reduction of mitochondrial mass in different loss of mortalin function models suggests that the increase in mitochondrial clearance is effective and represents an initial compensatory mechanism to maintain organellar quality.

Previously identified genetic defects in Parkin and PINK1 contribute to $\mathrm{PD}$ pathogenesis based on their role in mitochondrial clearance via autophagy and implicated impaired organellar quality control. ${ }^{46}$ This, however, was typically seen in response to toxic insults (i.e., via CCCP) that interfere with mitochondrial homeostasis in vitro, so that the pathophysiological relevance of these observations in neurons in vivo still remains to be determined. ${ }^{42,47}$ We found that PD-associated loss of mortalin function caused a primary defect in intramitochondrial molecular quality control that causes characteristic changes in mitochondrial function and dynamics, leading to an increased autophagic activity with increased Parkin-mediated clustering of mitochondria. These mitochondria were subsequently cleared from the healthy mitochondrial pool, leading to reduced mitochondrial mass in different cellular in vitro and ex vivo models. No alterations in cell viability under basal conditions were observed in fibroblasts of the human carrier of the A476T mutation that serves as a model of chronic mortalin haploinsufficiency. However, A476T fibroblasts showed an increased susceptibility toward proteolytic perturbation, indicating compensatory mechanisms active over lifetime. Thus, we speculate that the chronic impairment of the organellar quality control machinery sensitizes cells toward apoptotic cell death (Figure 8).

Indeed, overexpression of Parkin and PINK1 in mortalin knockdown models was able to revert mitochondrial

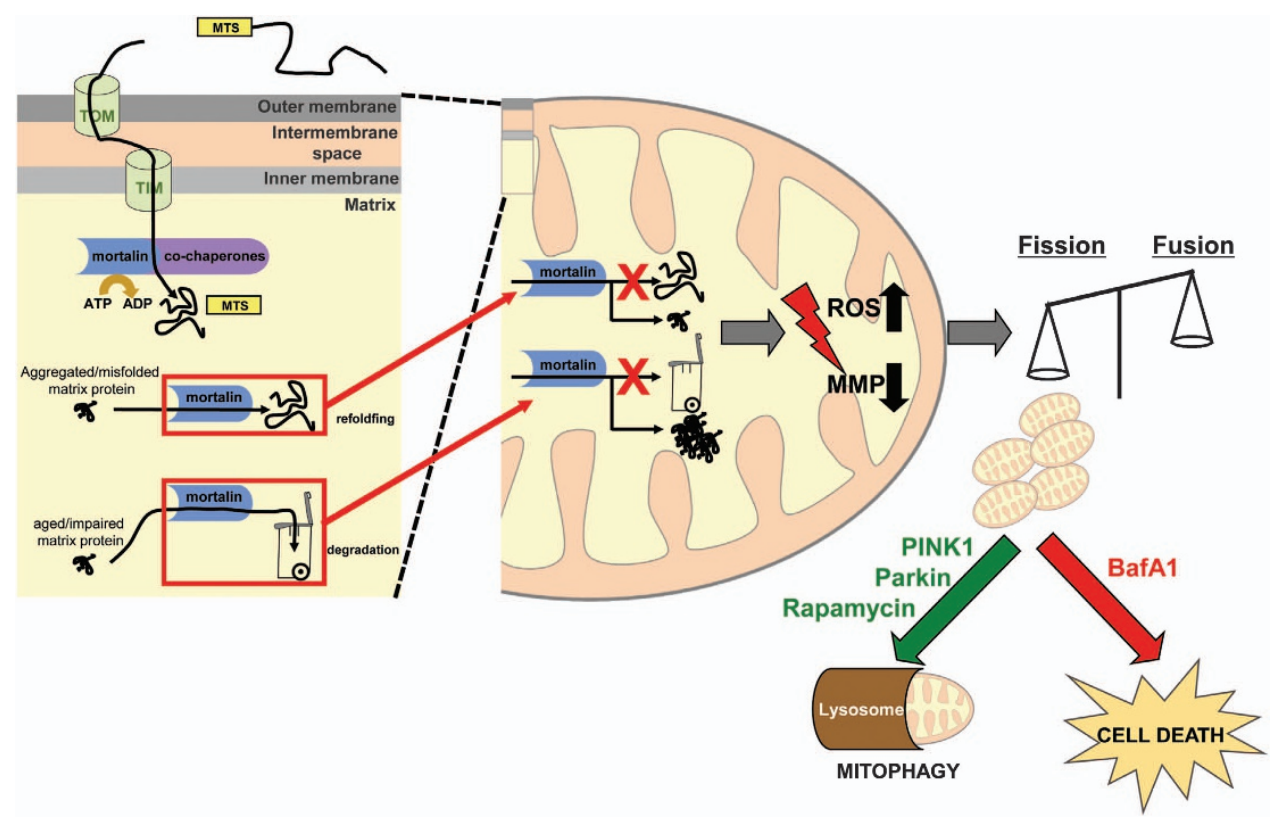

Figure 8 Overview of the consequences of loss of mortalin function in human cells. A schematic view highlights the consequences of loss of mortalin function found in different in vitro and in vivo models. Under physiological conditions, the mitochondrial chaperone mortalin is located within the mitochondrial matrix where it represents the only ATPase component of the import machinery and is responsible for the maintenance of mitochondrial function. It fulfils an active role in the effective import as well as the proper folding of nuclear encoded matrix proteins with the support of co-chaperones. Furthermore, mortalin occupies refolding capacity to deal with aggregated or misfolded proteins and acts as part of the mitochondrial quality control system for aged or impaired matrix proteins. Loss of mortalin function does not have a negative effect on effective import of mortalin into mitochondria or the interaction with other essential substrates. Hence, we consider the loss of protein function rather results in an impairment of the refolding or degradation capacity of mortalin. Consequently, instead of passing through the refolding process, aggregated/misfolded proteins remain defective. Similarly, aged/impaired proteins do not undergo the degradation process, but rather accumulate within the mitochondrial matrix. These alterations lead to increased ROS levels and impaired MMP, followed by changes in mitochondrial morphology. This is turn causes a shift in the normally well-balanced system of mitochondrial dynamics toward increased fission events. Both in vitro and in vivo, the loss of mortalin function correlates with a disequilibrium toward mitochondrial fission that either leads to lysosomal degradation of impaired mitochondria to maintain cellular integrity or may directly guide cell death. An inhibition of autophagy by use of the lysosomal inhibitor BafA1 (red arrow) in cells with reduced levels of mortalin exacerbates apoptosis, whereas either a genetic (PINK1 and Parkin overexpression) or pharmacological intervention (Rapamycin) enhances mitophagy so that dysfunctional mitochondria are removed from the mitochondrial pool (green arrow). Our results in different in vitro and ex vivo models support these effects upon loss of mortalin function either because of reduced levels of native mortalin protein or because of loss-of-function mutations in the mortalin gene 
phenotypes and reduce the susceptibility toward apoptotic cell death. This protective effect of PINK1 and Parkin was dependent on an intact autophagic machinery, as it was absent in fibroblasts from ATG5 KO mice that lack ATG5 as an essential factor for autophagosome formation. ${ }^{38}$ Similar protective effects on cells with reduced levels of mortalin were observed upon treatment with rapamycin, a compound known to induce autophagy by targeting mTORC $1 .{ }^{48}$ Therefore, our results suggest that effective removal of dysfunctional mitochondria via genetic (PINK1 and Parkin overexpression) or pharmacological intervention (rapamycin) may compensate mitochondrial phenotypes due to loss of mortalin.

The loss-of-function mechanism of PD-associated mortalin variants was suggested by an incomplete rescue of loss of mortalin-associated mitochondrial phenotypes by mutant R126W, A476T and P509S mortalin ${ }^{11}$ compared with wt mortalin in vitro. Furthermore, characteristic mitochondrial alterations in fibroblasts from the carrier of the A476T variant were observed ex vivo. Interestingly, a recent in vitro study in yeast also supported a loss of chaperone function for the PD-associated mortalin variants R126W and P509S using biochemical assays with purified recombinant proteins and a functional complementation assays in yeast. ${ }^{49} \mathrm{We}$ also performed experiments to rescue the inviable yeast $\mathrm{mH} H \mathrm{sp} 70$ (Ssc1)-null strain with either native Ssc1 or Ssc1 carrying the analogous PD-associated variants (R103W Ssc1 $=$ human R126W mortalin; P486S Ssc1 $\triangleq$ human P509S mortalin). We confirmed a severe growth defect for cells harboring the ATPase domain mutant R103W Ssc1 and consistently found no significant difference for cells complemented with P486S compared with native Ssc1 (see Supplementary Figure S6 and Goswami et al. ${ }^{49}$ ). Using A453T Ssc1 that corresponds to the A476T variant found in German PD patients, ${ }^{11}$ we found similar effects on growth as for the A486S Ssc1 variant, also located in the substrate-binding domain (see Supplementary Figure S6 and Goswami et al. ${ }^{49}$ ). Our findings support the notion of differential effects of $\mathrm{mtHsp} 70$ variants located in the ATPase domain or in the substrate-binding domain in yeast. Moreover, our results in human cellular in vitro and ex vivo models clearly show that the substrate-binding domain variants A476T and P509S exhibit a loss of mortalin function that translates into a phenotype of impaired mitochondrial proteostasis and changes in mitochondrial dynamics in human cells. These results underscore a concept of rare heterozygous variants in the essential mortalin gene that mediate neurodegeneration in PD via haploinsufficiency and are in line with the recent concepts on 'rare variants common disease' that were deduced from genomic approaches on neurological diseases and support the presence of rare variants with reduced penetrance contributing as risk factors to common disease (reviewed in Sharma et al. ${ }^{50}$ ). Recently, two novel missense variants (p.T333K and p.L358P) in the mortalin gene have been described that challenged the concept of mortalin variants in PD. For these variants, no alterations in different functional assays based on overexpression in SH-SY5Y cells were found. ${ }^{51}$ These results underscore that mortalin variants are not a frequent cause of PD and that functional consequences have to be carefully explored for all variants. Our results indicate that mortalin knockdown models in vitro or patient-based material ex vivo are useful to determine whether novel variants may be related to loss of mortalin function.

Based on the PD-associated symptoms of mortalin knockdown in Drosophila melanogaster in vivo, ${ }^{23}$ together with the concept of haploinsufficiency for disease-associated human variants in vitro, we suggest that an age-related decline of chaperoning capacities and subsequent collapse of protein homeostasis (reviewed in Baker et al. ${ }^{52}$ ) may be responsible for neurodegeneration and would be in line with aging as the most important risk factor for PD. ${ }^{53}$

Any mutation or insult lowering mitochondrial quality control capacity is predicted to preferentially affect neurons because of their increased requirement for energy and increased risk for accumulating mitochondrial damage. It is known that mitochondria in neurons exhibit a higher organellar half-life compared with other postmitotical tissues. ${ }^{54,55}$ One reason for the particular susceptibility of dopaminergic neurons toward loss of mortalin function may be because of a reduced mitochondrial mass of DA neurons in the substantia nigra compared with other neuronal populations as has been shown in mice. ${ }^{56}$ Translated into the human situation, the limited equipment of DA neurons in terms of mitochondria could argue in favor of an increased vulnerability of the cellular energy supply toward impaired mitochondrial homeostasis, a hypothesis that is supported by increasing evidence for an involvement of the PINK1/Parkin-mediated organellar quality control in PD. ${ }^{43,57}$ Moreover, neurons of the substantia nigra per se apparently exhibit a reduced mitophagic capacity that, in addition, may contribute to the selective vulnerability of DA neurons in PD. ${ }^{58}$ We have shown in this work that (1) a loss of mortalin function is sufficient to induce intramitochondrial proteolytic stress, (2) the autophagic machinery is activated in conditions of loss of mortalin function and (3) Parkin directly enhances the clustering of mitochondria in cells with reduced mortalin levels (Figure 7d). Parkin is part of an important step of the autophagic clearance machinery of damaged mitochondria from the cell. Therefore, overexpression of Parkin in human and murine models of loss of mortalin function may help to more efficiently remove dysfunctional mitochondria. This would further revert the altered mitochondrial morphology and protect from cell death (Figure $6 \mathrm{~d}$ and Figures $7 \mathrm{a}-\mathrm{c}$ ). Indeed, rescue experiments including both PINK1 and Parkin point to a protective role of the PINK1/Parkin pathway being essential for removal of dysfunctional mitochondria as a result of mortalin loss of function.

In summary, our study emphasizes the importance of mortalin for mitochondrial proteostasis and underscores the relevance of impaired protein quality control within the mitochondria in initiating a cascade of events leading to cell death. Our findings further stage mortalin-mediated disruption of mitochondrial integrity into the context of Parkin- and PINK1-associated PD phenotypes.

\section{Materials and Methods}

Cloning of wt and mutant mortalin. Cloning of mortalin CDNA and its mutagenesis for insertion of the R126W, A476T and P509S variants has been described previously. ${ }^{11}$

Cell culture, transfection and generation of stable cell lines. Skin biopsies were taken from one offspring of a PD patient, both carrying the heterozygous A476T variant in the mortalin gene, and an unaffected sibling 
control. The carrier of the A476T variant did not show signs of PD at that time. All biopsies were obtained with patient's consent and approval of the local ethics committee.

Cell culture of SH-SY5Y and HEK293 cells and human fibroblasts has been described previously. ${ }^{11}$ The m5-7 MEFs were prepared to achieve a knockout of ATG5 as previously described. ${ }^{37}$ Passage number of fibroblasts was $<10$ for all experiments. Only fibroblasts with the same passage number were taken.

Western blot. Cell lysis was carried out identically for SH-SY5Y cells, HEK293 cells and human fibroblasts as well as MEFs. Cells were washed with phosphatebuffered saline (PBS) and detached with Trypsin at $37^{\circ} \mathrm{C}$ for $3 \mathrm{~min}$. After $5 \mathrm{~min}$ of centrifugation at 14000 r.p.m., cells were lysed in buffer containing $0.1 \%$ Triton $\mathrm{X}-100$ in PBS and $1 \times$ protease inhibitor cocktail (Roche, Penzberg, Germany). Proteins were detected by using antibodies against mortalin (anti-GRP75, Santa Cruz Biotechnology, Dallas, TX, USA), $\beta$-Actin (Sigma-Aldrich, Munich, Germany), GAPDH (Life Technologies, Carlsbad, CA, USA), Tom20 (Santa Cruz Biotechnology), Hsp60 (from A Azem, Tel-Aviv University, Ramat Aviv, Israel), activated caspase 3 (Cell Signaling Technology, Danvers, MA, USA), activated caspase 9 (Cell Signaling Technology), Flag (Sigma-Aldrich), $\alpha$-tubulin (Sigma-Aldrich), $\beta$-Tubulin (anti- $\beta$-Tubulin, (E7), Developmental Studies Hybridoma Bank, University of lowa, lowa City, IA, USA), GAPDH (anti-GAPDH (G9545), Sigma-Aldrich) and rodent OXPHOS (no. MS604 Mitoscience (Abcam), Cambridgeshire, UK). Secondary antibodies were purchased from GE Healthcare (Buckinghamshire, UK)

Densitometry from western blot was performed using the ImageJ 1.410 software (Wayne Rasband; National Institutes of Health, Bethesda, MD, USA).

Preparation of mitochondrial fractions. Mitochondria were purified from tetracycline-induced (nontargeting or mortalin miRNA) knockdown SH-SY5Y cells in homogenization buffer containing $10 \mathrm{mM} \mathrm{HEPES} \mathrm{(pH} \mathrm{7.4),} 50 \mathrm{mM}$ sucrose, $0.4 \mathrm{M}$ mannitol, $10 \mathrm{mM} \mathrm{KCL}$ and $1 \mathrm{mM}$ EGTA, using a two-step differential centrifugation at $600 \times g$ and $3000 \times g$, respectively, for $15 \mathrm{~min}$ at $4^{\circ} \mathrm{C}$. The mitochondria-enriched fraction was resuspended in a buffer containing $20 \mathrm{mM}$ HEPES (pH 7.4), $0.4 \mathrm{M}$ mannitol, $10 \mathrm{mM} \mathrm{NaH}_{2} \mathrm{PO}_{4}$ and $0.5 \mathrm{mM}$ EGTA to equal protein concentration of $1 \mu \mathrm{g} / \mu \mathrm{l}$. Equal amounts of this mitochondrial fraction were added to equal volumes of a lysis buffer containing $4 \%(\mathrm{w} / \mathrm{v})$ SDS or $2 \%(\mathrm{v} / \mathrm{v})$ NP40. Proteins were resolved in NuPAGE $4-12 \%$ gradient gels (Life Technologies) and visualized by silver staining or for western blot analysis using the indicated antibodies as described in Moisoi et al..$^{59}$

Silver stain of soluble and insoluble mitochondrial fractions. NuPAGE gradient gels were first placed in $10 \%$ (v/v) ethanol for $10 \mathrm{~min}$ on a shaker and then in $1 \%(\mathrm{v} / \mathrm{v}) \mathrm{HNO}_{3}$ (Roth, Karlsruhe, Germany) for $15 \mathrm{~min}$ before incubation with $0.2 \%(\mathrm{w} / \mathrm{v}) \mathrm{AgNO}_{3}$ (Sigma-Aldrich) for $30 \mathrm{~min}$. After a short wash with $\mathrm{ddH}_{2} \mathrm{O}$, bands were made visible with $3 \%$ (w/v) $\mathrm{Na}_{2} \mathrm{CO}_{3}$ (Roth) with $50 \mu \mathrm{l}$ formaldehyde solution (Sigma-Aldrich) per $100 \mathrm{ml}$. The reaction was stopped with $10 \%$ (v/v) acetic acid (Merck, Darmstadt, Germany) for 2 min when the staining was sufficient and gels were kept in $\mathrm{ddH}_{2} \mathrm{O}$ until digitalization.

Mitonuclear imbalance. The mitochondrial SDS fraction of equalized lysates (described above) were resolved on NuPAGE 4-12\% gradient gels (Life Technologies) and western blots probed for total rodent OXPHOS (AbCam 110413, MS604) that contains antibodies against ATP5A (nuclear encoded) and MTCO1 (mitochondrially encoded) according to Houtkooper et al. ${ }^{26}$ Tom20 was used as an additional loading control (Santa Cruz Biotechnology). A representative western blot is shown. Densitometry of each band from three independent experiments was calculated using ImageJ and the mitonuclear imbalance expressed as a ratio of proteins expressed from nuclear DNA to mitochondrial DNA according to Houtkooper et al. ${ }^{26}$

RNA interference-mediated downregulation of mortalin and overexpression of DNA plasmids. Mortalin knockdown was achieved by transfection of Flexitube siRNA (Hs_HSPA9_1, 3'-AlexaFluor555) or chemically unmodified nontargeting control siRNA (AllStars Negative siRNA AF 555) from Qiagen (Hilden, Germany) into SH-SY5Y cells. A total of 70000 cells were transfected with $160 \mathrm{nM}$ of siRNA or negative control siRNA using HiPerFect transfection reagent (Qiagen), leading to a reduction in mortalin protein of $>70 \%$ in cells transfected with mortalin siRNA compared with control cells (Supplementary Figure S1A).
For mortalin knockdown using a tetracycline-inducible miRNA system (BLOCK IT, Life Technologies), miRNA sequences were designed targeting two regions on the mortalin gene (nucleotide start sites 773 and 1939) that were chained together and under polycistronic control of the same promoter in the same pcDNA 6.2-GW/ EmGFP-miR miRNA vector backbone. Induced expression was monitored by EmGFP fluorescence (EmGFP-miR vector, Life Technologies) and by western blot. Additional transfection of any plasmid was achieved using HiPerFect transfection reagent.

Immunofluorescence microscopy upon transient transfection of siRNAs. For immunocytochemistry, cells were seeded on collagen-coated slides. For successful knockdown of mortalin, siRNA was transfected $5 \mathrm{~h}$ after seeding the cells. GFP-LC3 and respective plasmids were transfected in the ratio of $1: 4$ using HiPerFect transfection reagent $12 \mathrm{~h}$ after siRNA treatment to control for positive transfection. Cells positive for GFP-LC3 were considered as also harboring the other plasmid. Analysis of the number of GFP-LC3 puncta per cell was done via an unbiased automatical counting approach using ImageJ software.

For colocalization and morphology studies, reintroduction of any plasmid was similarly applied $12 \mathrm{~h}$ after siRNA treatment. For OTC/dOTC experiments, the plasmid was transfected $5 \mathrm{~h}$ after seeding the cells. In this condition, siRNA was applied $12 \mathrm{~h}$ after plasmid transfection. If needed for rescue experiments, wt mortalin or empty vector were cotransfected with empty pEGFP-N1 plasmid in the ratio of $1: 4$ to control for successful plasmid transfection. Fixation of cells occurred $24 \mathrm{~h}$ after the transfection procedure.

To chemically elicit a mitochondrial unfolded stress response, cells were treated with $0.25 \mu \mathrm{M}$ of the $\mathrm{Hsp} 90$ inhibitor 17-AAG for $30 \mathrm{~h}$ before fixation. To induce ER stress leading to $\mathrm{Ca}^{2+}$ depletion, cells were treated with $30 \mu \mathrm{M}$ for $5 \mathrm{~h}$. Lysosomal inhibition was evoked by treatment with $100 \mathrm{nM}$ BafA 1 for $1 \mathrm{~h}$ followed by incubation in normal medium for $4 \mathrm{~h}$. BafA1 prevents the fusion between autophagosomes and lysosomes and is an established method to assess the autophagic flux. For activation of autophagy, cells were incubated with $100 \mathrm{nM}$ Rapamycin for $24 \mathrm{~h}$.

Fixation procedure and microscopic analysis were performed as described previously. ${ }^{11}$ If needed, cells were incubated for $15 \mathrm{~min}$ in $100 \mathrm{nM}$ Lyostracker Red DND-99 (Life Technologies) before fixation. Proteins were detected by using antibodies against mortalin (anti-GRP75, Santa Cruz Biotechnology), Tom20 (Santa Cruz Biotechnology), Lamp-1 (Hybridoma Bank of lowa University), cleaved Caspase-3 (Cell Signaling Technology) and Flag (Sigma-Aldrich). Secondary antibodies were purchased from Life Technologies or Zymed (San Francisco, CA, USA). Hoechst 33342 (Life Technologies) was used to stain nuclei.

Images were analyzed by Zeiss software AxioVision 4.6 (Zeiss, Jena, Germany) and ImageJ 1.410 software.

Immunofluorescence microscopy analysis of endogenous WIPI-2 puncta formation and endogenous p62 localization. To assess endogenous WIPI-2 puncta formation, SH-SY5Y cells with inducible expression of miRNA targeting mortalin were seeded on coverslips in 24-well plates ( 50000 cells per well). Mortalin miRNA expression was induced for 24,48 and $72 \mathrm{~h}$ using $1 \mu \mathrm{g} / \mathrm{ml}$ tetracycline, followed by a 3-h incubation in starvation medium (Earle's balanced salt solution (EBSS)) including BafA1 (200 nM). Subsequently, cells were fixed in $4 \%$ paraformaldehyde and indirect immunofluorescence conducted as previously described ${ }^{34}$ using anti-WIPI2 antibodies (1:50, Abgent, San Diego, CA, USA) and anti-lgG-Alexa546 (1:200, Life Technologies). Using confocal microscopy (Zeiss LSM 510) and ImageJ analysis, the number of WIPI-2 puncta per cell as well as fluorescent puncta area was determined.

To assess endogenous p62 localization, SH-SY5Y cells with inducible expression of miRNA targeting mortalin were seeded in 96-well plates (10000 cells per well) and grown in tetracycline-containing medium for $72 \mathrm{~h}$ followed by a 3-h treatment with control medium (CM) or EBSS in the presence or absence of BafA1 include concentration of BafA (200nM). Cells were fixed in $4 \%$ paraformaldehyde and indirect immunofluorescence conducted as previously described $^{34}$ using anti-p62 antibodies (1:50, Santa Cruz, Dallas, TX, USA) and anti-lgG-Alexa546 (1:200, Life Technologies) and DAPI to stain cell nuclei. Using an automated image acquisition and analysis platform, p62 inclusions (puncta) per cell were imaged and analyzed from up to 5000 cells as previously described. ${ }^{37}$

Parkin recruitment studies. CCCP and Valinomycin are the only known inducers of Parkin-mediated mitophagy, ${ }^{42,43}$ yet there is a lack of accepted methods to assess Parkin translocation following induction with other toxins such as rotenone. Therefore, we assessed the intermediate clustering of mitochondria preceding mitochondrial removal. ${ }^{42}$ We knocked down mortalin using an inducible miRNA system in HeLa cells that are devoid of endogenous Parkin. ${ }^{42}$ Exogenous 
myc-Parkin was introduced and the mitochondria in cells positive for miRNA only or miRNA + myc-Parkin were analyzed for number per cell using ImageJ (threshold 18-infinity and number of mitochondrial particles).

Live cell imaging microscopy. Live cell imaging was assessed to study mitochondrial morphology and mass as well as colocalization of mitochondria with lysosomes. Cells were cultured in Lab-TekHII chambered coverglasses (155382; NalgeNunc International, Penfield, NY, USA).

For mitochondrial morphology and mass as well as colocalization studies, mitochondria were visualized by $100 \mathrm{nM}$ MitoTracker Green FM (Life Technologies), lysosomes by $100 \mathrm{nM}$ Lyostracker Red DND-99 (Life Technologies) and nuclei by Hoechst 33342 after an incubation time of $15 \mathrm{~min}$. In studies using Lyostracker Red DND-99, the dye was also present within the medium during imaging the cells for improved visualization of lysosomes. Live cell imaging analysis was performed as described previously. ${ }^{11}$ The series of images were saved uncompressed and analyzed with AxioVision software (Zeiss) and ImageJ 1.410 software, respectively.

Analysis of morphology and mass of mitochondria. Analysis of mitochondrial morphology was done as described previously. Morphological characteristics such as form factor, aspect ratio, area circularity and mitochondrial mass were evaluated using ImageJ software. To analyze the mitochondrial mass, the total area of mitochondria per single cell was calculated.

Fluorescence-activated cell sorting. Briefly, SH-SY5Y cells were washed with PBS at room temperature and then incubated in either Annexin $\mathrm{V}$ binding buffer (Biolegend, San Diego, CA, USA) or the same buffer containing Annexin V-Pacific blue (BioLegend, according to the manufacturer's titration) for 15 min on ice. Cells were sorted on a CyAn ADP (Beckman Coulter, Brea, CA, USA) FACS machine according to their Annexin V-Pacific Blue signal above the precontrolled threshold set by the unstained control cells. The percent of cells in the population above this threshold was also gated for those cells containing control siRNA-647 or Mortalin siRNA-647 fluorescent signal.

LC3 lipidation. To assess endogenous enogenous LC3 lipidation, SH-SY5Y cells with inducible expression of miRNA targeting mortalin were seeded in 24-well plates (50000 cells per well). Mortalin miRNA expression was induced for $72 \mathrm{~h}$ using $1 \mu \mathrm{g} / \mathrm{ml}$ tetracycline, followed by a 3-h incubation in control medium or starvation medium (EBSS) including BafA1 (200 nM) or not. Subsequently, cells were lysed in hot Laemmli buffer and resolved by SDS-PAGE. Using LC3 antibodies (Nanotools, Munich, Germany), unconjugated LC3 (LC3-I) and PE-conjugated LC3 (LC3-II) were detected by standard ECL procedures and PeqLab Fusion SL (Erlangen, Germany).

Statistical analysis. The imaging data were analyzed using Student's t-test; all the statistical tests were nonpaired two sided and those with a $P$-value of $<0.05$ were considered to be statistically significant. Data are expressed as mean \pm S.E. values $\left({ }^{\star} P<0.05 ;{ }^{* \star} P<0.01 ;{ }^{* \star *} P<0.001\right)$.

\section{Conflict of Interest}

The authors declare no conflict of interest.

Acknowledgements. We thank Noboru Mizushima, University of Tokio, Japan, for providing the m5-7 cells, Tamotsu Yoshimori, Osaka University, Japan, for providing the GFP-LC3 plasmid and Sven Geisler, Hertie-Institute for Clinical Brain Research, University of Tübingen, for invaluable discussions on the mitochondrial clustering experiments. This work was supported by grants from the Fritz Thyssen Foundation (10.11.2.153 to RK and 10.12.1.192 to TMR); the German Research Council (DFG, KR2119/8-1 to RK, RA 1804/2-1 to TMR, RA1028/4-1 to DR, SFB 773TP A03 to TP-C); the Federal Ministry for Education and Research (BMBF, NGFNplus; 01GS08134 to RK and OR); and by a doctoral scholarship from the charitable Hertie Foundation (to LFB).

1. Sharma M, loannidis JP, Aasly JO, Annesi G, Brice A, Van Broeckhoven C et al. Large-scale replication and heterogeneity in Parkinson disease genetic loci. Neurology 2012; 79: 659-667.

2. Burbulla LF, Kruger R. Converging environmental and genetic pathways in the pathogenesis of Parkinson's disease. J Neurol Sci 2011; 306: 1-8.
3. Mandemakers W, Morais VA, De Strooper B. A cell biological perspective on mitochondrial dysfunction in Parkinson disease and other neurodegenerative diseases. J Cell Sci 2007; 120: $1707-1716$.

4. Schapira AH. Mitochondrial dysfunction in neurodegenerative diseases. Neurochem Res 2008; 33: 2502-2509.

5. Palacino JJ, Sagi D, Goldberg MS, Krauss S, Motz C, Wacker M et al. Mitochondrial dysfunction and oxidative damage in parkin-deficient mice. J Biol Chem 2004; 279: 18614-18622.

6. Valente EM, Abou-Sleiman PM, Caputo V, Muqit MM, Harvey K, Gispert S et al. Hereditary early-onset Parkinson's disease caused by mutations in PINK1. Science 2004; 304: $1158-1160$.

7. Krebiehl G, Ruckerbauer S, Burbulla LF, Kieper N, Maurer B, Waak J et al. Reduced basal autophagy and impaired mitochondrial dynamics due to loss of Parkinson's diseaseassociated protein DJ-1. PLoS One 2010; 5: e9367.

8. Jin J, Li GJ, Davis J, Zhu D, Wang Y, Pan C et al. Identification of novel proteins associated with both alpha-synuclein and DJ-1. Mol Cell Proteomics 2007; 6: 845-859.

9. Davison EJ, Pennington K, Hung CC, Peng J, Rafiq R, Ostareck-Lederer A et al. Proteomic analysis of increased Parkin expression and its interactants provides evidence for a role in modulation of mitochondrial function. Proteomics 2009; 9: 4284-4297.

10. Rakovic A, Grünewald A, Voges L, Hofmann S, Orolicki S, Lohmann K et al. PINK1interacting proteins: proteomic analysis of overexpressed PINK1. Parkinsons Dis 2011; 2011: 153979.

11. Burbulla LF, Schelling $C$, Kato H, Rapaport D, Woitalla D, Schiesling $C$ et al. Dissecting the role of the mitochondrial chaperone mortalin in Parkinson's disease: functional impact of disease-related variants on mitochondrial homeostasis. Hum Mol Genet 2010; 19: 4437-4452.

12. Wadhwa R, Pereira-Smith OM, Reddel RR, Sugimoto Y, Mitsui Y, Kaul SC. Correlation between complementation group for immortality and the cellular distribution of mortalin. Exp Cell Res 1995; 216: 101-106.

13. Webster TJ, Naylor DJ, Hartman DJ, Hoj PB, Hoogenraad NJ. cDNA cloning and efficient mitochondrial import of pre-mtHSP70 from rat liver. DNA Cell Biol 1994; 13: 1213-1220.

14. Rehling $P$, Brandner K, Pfanner N. Mitochondrial import and the twin-pore translocase. Nat Rev Mol Cell Biol 2004; 5: 519-530.

15. Schneider HC, Berthold J, Bauer MF, Dietmeier K, Guiard B, Brunner M et al. Mitochondrial Hsp70/MIM44 complex facilitates protein import. Nature 1994; 371: 768-774.

16. Deocaris CC, Kaul SC, Wadhwa R. On the brotherhood of the mitochondrial chaperones mortalin and heat shock protein 60. Cell Stress Chaperones 2006; 11: 116-128.

17. Yaguchi T, Aida S, Kaul SC, Wadhwa R. Involvement of mortalin in cellular senescence from the perspective of its mitochondrial import, chaperone, and oxidative stress management functions. Ann NY Acad Sci 2007; 1100: 306-311.

18. Liu Y, Liu W, Song XD, Zuo J. Effect of GRP75/mthsp70/PBP74/mortalin overexpression on intracellular ATP level, mitochondrial membrane potential and ROS accumulation following glucose deprivation in PC12 cells. Mol Cell Biochem 2005; 268: 45-51.

19. Jin J, Hulette C, Wang Y, Zhang T, Pan C, Wadhwa R et al. Proteomic identification of a stress protein, mortalin/mthsp70/GRP75: relevance to Parkinson disease. Mol Cell Proteomics 2006; 5: 1193-1204.

20. Kaul SC, Deocaris CC, Wadhwa R. Three faces of mortalin: a housekeeper, guardian and killer. Exp Gerontol 2007; 42: 263-274.

21. Kaul SC, Yaguchi T, Taira K, Reddel RR, Wadhwa R. Overexpressed mortalin (mot-2)/ mthsp70/GRP75 and hTERT cooperate to extend the in vitro lifespan of human fibroblasts. Exp Cell Res 286: 96-1012003.

22. Shi M, Jin J, Wang Y, Beyer RP, Kitsou E, Albin RL et al. Mortalin: a protein associated with progression of Parkinson disease? J Neuropathol Exp Neurol 2008; 67: 117-124.

23. Zhu JY, Vereshchagina N, Sreekumar V, Burbulla LF, Costa AC, Daub KJ et al. Knockdown of Hsc70-5/mortalin induces loss of synaptic mitochondria in a Drosophila Parkinson's disease model. PLoS One 2013; 8: e83714.

24. Park J, Lee SB, Lee S, Kim Y, Song S, Kim S et al. Mitochondrial dysfunction in Drosophila PINK1 mutants is complemented by parkin. Nature 2006; 441: 1157-1161.

25. Clark IE, Dodson MW, Jiang C, Cao JH, Huh JR, Seol JH et al. Drosophila pink1 is required for mitochondrial function and interacts genetically with parkin. Nature 2006; 441 : 1162-1166.

26. Houtkooper RH, Mouchiroud L, Ryu D, Moullan N, Katsyuba E, Knott G et al. Mitonuclear protein imbalance as a conserved longevity mechanism. Nature 2013; 497: 451-457.

27. Broadley SA, Hartl FU. Mitochondrial stress signaling: a pathway unfolds. Trends Cell Biol 2008; 18: 1-4.

28. Pimenta de Castro I, Costa AC, Lam D, Tufi R, Fedele V, Moisoi N et al. Genetic analysis of mitochondrial protein misfolding in Drosophila melanogaster. Cell Death Differ 2012; 19: 1308-1316.

29. Zhao Q, Wang J, Levichkin IV, Stasinopoulos S, Ryan MT, Hoogenraad NJ. A mitochondrial specific stress response in mammalian cells. EMBO J2002; 21: 4411-4419.

30. Kieper N, Holmström KM, Ciceri D, Fiesel FC, Wolburg H, Ziviani E et al. Modulation of mitochondrial function and morphology by interaction of $\mathrm{Omi} / \mathrm{HtrA} 2$ with the mitochondrial fusion factor OPA1. Exp Cell Res 2010; 316: 1213-1224.

31. Burbulla LF, Krebiehl G, Kruger R. Balance is the challenge-the impact of mitochondrial dynamics in Parkinson's disease. Eur J Clin Invest 2010; 40: 1048-1060.

32. Kabeya Y, Mizushima N, Ueno T, Yamamoto A, Kirisako T, Noda T et al. LC3, a mammalian homologue of yeast Apg8p, is localized in autophagosome membranes after processing. EMBO J 2000; 19: 5720-5728. 
33. Klionsky DJ, Abdalla FC, Abeliovich $\mathrm{H}$, Abraham RT, Acevedo-Arozena A, Adeli K et al. Guidelines for the use and interpretation of assays for monitoring autophagy. Autophagy 2012; 8: 445-544.

34. Proikas-Cezanne T, Waddell S, Gaugel A, Frickey T, Lupas A, Nordheim A. WIPI-1alpha (WIPI49), a member of the novel 7-bladed WIPI protein family, is aberrantly expressed in human cancer and is linked to starvation-induced autophagy. Oncogene 2004; 23: 9314-9325

35. Codogno P, Mehrpour M, Proikas-Cezanne T. Canonical and non-canonical autophagy: variations on a common theme of self-eating? Nat Rev Mol Cell Biol 2012; 13: 7-12.

36. Proikas-Cezanne $\mathrm{T}$, Robenek $\mathrm{H}$. Freeze-fracture replica immunolabelling reveals human WIPI-1 and WIPI-2 as membrane proteins of autophagosomes. J Cell Mol Med 2011; 15 : 2007-2010.

37. Mauthe M, Jacob A, Freiberger S, Hentschel K, Stierhof YD, Codogno P et al. Resveratrolmediated autophagy requires WIPI-1-regulated LC3 lipidation in the absence of induced phagophore formation. Autophagy 2011; 7: 1448-1461.

38. Hosokawa N, Hara Y, Mizushima N. Generation of cell lines with tetracycline-regulated autophagy and a role for autophagy in controlling cell size. FEBS Lett 2006; 580 2623-2629.

39. Kang BH, Plescia J, Dohi T, Rosa J, Doxsey SJ, Altieri DC. Regulation of tumor cell mitochondrial homeostasis by an organelle-specific Hsp90 chaperone network. Cell 2007; 131: 257-270.

40. Margineantu DH, Emerson CB, Diaz D, Hockenbery DM. Hsp90 inhibition decreases mitochondrial protein turnover. PLoS One 2007; 2: e1066.

41. Zhao P, Xiao X, Kim AS, Leite MF, Xu J, Zhu X et al. c-Jun inhibits thapsigargin-induced ER stress through up-regulation of DSCR1/Adapt78. Exp Biol Med 2008; 233: 1289-1300.

42. Geisler S, Holmström KM, Skujat D, Fiesel FC, Rothfuss OC, Kahle PJ et al. PINK1/ Parkin-mediated mitophagy is dependent on VDAC1 and p62/SQSTM1. Nat Cell Biol 2010; 12: 119-131.

43. Narendra D, Tanaka A, Suen DF, Youle RJ. Parkin-induced mitophagy in the pathogenesis of Parkinson disease. Autophagy 2009; 5: 706-708.

44. Antony PM, Diederich NJ, Kruger R, Balling R. The hallmarks of Parkinson's disease. FEBS J 2013; 280: 5981-5993.

45. Chen Y, Dorn GW 2nd. PINK1-phosphorylated mitofusin 2 is a Parkin receptor for culling damaged mitochondria. Science 2013; 340: 471-475

46. Narendra DP, Jin SM, Tanaka A, Suen DF, Gautier CA, Shen J et al. PINK1 is selectively stabilized on impaired mitochondria to activate Parkin. PLOS Biol 2010; 8: e1000298.

47. Vives-Bauza C, Zhou C, Huang Y, Cui M, de Vries RL, Kim J et al. PINK1-dependen recruitment of Parkin to mitochondria in mitophagy. Proc Natl Acad Sci USA 2010; 107: 378-383
48. Sarkar S, Rubinsztein DC. Small molecule enhancers of autophagy for neurodegenerative diseases. Mol Biosyst 2008; 4: 895-901.

49. Goswami AV, Samaddar M, Sinha D, Purushotham J, D'Silva P. Enhanced J-protein interaction and compromised protein stability of $\mathrm{mtHsp} 70$ variants lead to mitochondrial dysfunction in Parkinson's disease. Hum Mol Genet 2012; 21: 3317-3332.

50. Sharma M, Kruger R, Gasser T. From genome-wide association studies to next-generation sequencing: lessons from the past and planning for the future. JAMA Neurol 2013; 71: 5-6.

51. Freimann K, Zschiedrich K, Brüggemann N, Grünewald A, Pawlack H, Hagenah J et al. Mortalin mutations are not a frequent cause of early-onset Parkinson disease. Neurobiol Aging 2013; 34: 2694 e19-20.

52. Baker MJ, Tatsuta T, Langer T. Quality control of mitochondrial proteostasis. Cold Spring Harb Perspect Biol 2011; 3: 1-19.

53. Hindle JV. Ageing, neurodegeneration and Parkinson's disease. Age Ageing 2010; 39: 156-161.

54. Menzies RA, Gold PH. The turnover of mitochondria in a variety of tissues of young adult and aged rats. J Biol Chem 1971; 246: 2425-2429

55. O'Toole M, Latham R, Baqri RM, Miller KE. Modeling mitochondrial dynamics during in vivo axonal elongation. J Theor Biol 2008; 255: 369-377.

56. Liang CL, Wang TT, Luby-Phelps K, German DC. Mitochondria mass is low in mouse substantia nigra dopamine neurons: implications for Parkinson's disease. Exp Neurol 2007; 203: $370-380$

57. Wang X, Winter D, Ashrafi G, Schlehe J, Wong YL, Selkoe D et al. PINK1 and Parkin target Miro for phosphorylation and degradation to arrest mitochondrial motility. Cell 2011; 147: 893-906.

58. Diedrich M, Kitada T, Nebrich G, Koppelstaetter A, Shen J, Zabel C et al. Brain region specific mitophagy capacity could contribute to selective neuronal vulnerability in Parkinson's disease. Proteome Sci 2011; 9: 59.

59. Moisoi N, Klupsch K, Fedele V, East P, Sharma S, Renton A et al. Mitochondria dysfunction triggered by loss of $\mathrm{HtrA} 2$ results in the activation of a brain-specific transcriptional stress response. Cell Death Differ 2009; 16: 449-464.

(c) (1) () $\Theta$ Cell Death and Disease is an open-access journal published by Nature Publishing Group. This work is licensed under a Creative Commons Attribution-NonCommercialNoDerivs 3.0 Unported License. To view a copy of this license, visit http://creativecommons.org/licenses/by-nc-nd/3.0/

Supplementary Information accompanies this paper on Cell Death and Disease website (http://www.nature.com/cddis) 